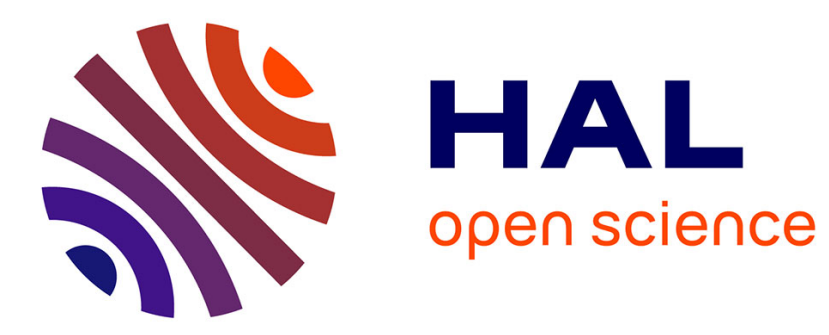

\title{
Caractérisation et propriétés physiques des défauts induits dans les transistors MOS submicroniques par injections de porteurs chauds
}

Dominique Vuillaume

\section{- To cite this version:}

Dominique Vuillaume. Caractérisation et propriétés physiques des défauts induits dans les transistors MOS submicroniques par injections de porteurs chauds. Journal de Physique III, 1992, 2 (5), pp.777804. 10.1051/jp3:1992159 . jpa-00248781

HAL Id: jpa-00248781

https://hal.science/jpa-00248781

Submitted on 1 Jan 1992

HAL is a multi-disciplinary open access archive for the deposit and dissemination of scientific research documents, whether they are published or not. The documents may come from teaching and research institutions in France or abroad, or from public or private research centers.
L'archive ouverte pluridisciplinaire HAL, est destinée au dépôt et à la diffusion de documents scientifiques de niveau recherche, publiés ou non, émanant des établissements d'enseignement et de recherche français ou étrangers, des laboratoires publics ou privés. 
Classification

Physics Abstracts

$73.400-73.60 \mathrm{H}$

\title{
Caractérisation et propriétés physiques des défauts induits dans les transistors MOS submicroniques par injections de porteurs chauds
}

\author{
Dominique Vuillaume \\ (1) Institut d'Electronique et de Microélectronique du Nord (IEMN), UMR 9929, CNRS, 41 \\ boulevard Vauban, 59046 Lille Cedex, France
}

(Reçu le 28 novembre 1991, accepté le 4 février 1992)

\begin{abstract}
Résumé. - La fiabilité des technologies CMOS submicroniques est un enjeu important pour le développement des circuits ULSI des prochaines années. Nous présentons une revue de nos récents travaux sur la caractérisation des propriétés physiques des défauts induits dans les transistors MOS par l'injection de porteurs chauds. Le rôle de ces défauts dans la dégradation des performances des transistors est examiné avec soin et discuté par rapport à une revue des principaux résultats de la littérature. Nos contributions portent sur : i) l'utilisation de techniques fines (pompage de charge et mesure de courant de grille par une technique de « grille-flottante ») pour étudier les défauts créés dans l'oxyde et à l'interface $\mathrm{Si}^{-\mathrm{SiO}_{2}}$, ii) la mesure des propriétés optiques et électroniques de ces défauts d'oxyde conduisant à en préciser la nature et iii) une étude auto-cohérente des relations défauts-performances au moyen de simulations bidimensionnelles des composants MOSFET vieillis.
\end{abstract}

\begin{abstract}
The reliability of the submicrometer CMOS technologies is a key issue for the development of advanced ULSI devices and circuits. We present a review of our recent results on the characterization of the physical properties of hot-carrier-induced defects in MOS transistors. The role played by these defects in the device performance degradation is carefully studied and critically discussed in comparison with the main results given in the literature. Our contributions point out : i) the characterization of oxide and $\mathrm{Si}_{-} \mathrm{SiO}_{2}$ interface defects by powerful techniques (charge pumping and measurement of the gate current by "floating-gate " technique), ii) the measurements of the optical and electronic properties of these oxide defects leading to get insights on their microscopic nature and iii) a self-consistent study of the defect-performance relationship of the aged MOSFET devices through bidimensional devices simulations.
\end{abstract}

\section{Introduction.}

Le rôle des défauts ponctuels (i.e. de la taille de quelques distances interatomiques) dans les dispositifs métal-oxyde-semiconducteur (MOS) devient de plus en plus important au fur et à mesure de la réduction des dimensions des composants. La réalisation de ces dispositifs demande une complexité et un nombre d'étapes élémentaires de fabrication de plus en plus important. Le contrôle de l'introduction de défauts cristallographiques et d'impuretés (en 
particulier métalliques) est un problème crucial. Récemment, les mémoires DRAM 4 Mbit (techno $0,8 \mu \mathrm{m}$ ) ont atteint leur maturité comme produit de $\mathrm{ma}^{-c e}$, et les mémoires DRAM $16 \mathrm{Mbit}$ (techno $0,5 \mu \mathrm{m}$ ) sont en préparation pour prendre la reì a Des prototypes de mémoires DRAM $64 \mathrm{Mbit}$ (techno $0,3 \mu \mathrm{m}$ ) ont été réalisés avec succès [1]. Les caractéristiques électriques finales de ces composants sont de plus en plus sensibles aux imperfections et défauts des matériaux.

D'autre part, la réduction des longueurs de canaux et des épaisseurs d'oxyde de grille des MOSFET implique la présence de champs électriques importants dans les dispositifs MOSFET microniques et submicroniques. Cela rend primordiaux les aspects de fiabilitédégradation. Là aussi, les défauts sont responsables de ces problèmes, défauts à l'interface Si$\mathrm{SiO}_{2}$ et dans l'oxyde. Ces défauts sont générés à cause de l'existence de porteurs chauds (i.e. ayant acquis une énergie importante sous l'influence de champs électriques intenses) qui sont injectés dans l'oxyde et qui sont capables de dégrader la structure microscopique des matériaux (semiconducteur et oxyde) des dispositifs MOS.

Même dans le cas de dispositifs ultra-submicroniques fonctionnant avec des tensions d'alimentation très faibles $(2,5 \mathrm{~V}$ pour un MOSFET $0,3 \mu \mathrm{m})$ ces phénomènes de dégradation persistent alors qu'on aurait pu espérer leur disparition [2, 3]. En effet, à ces tensions de fonctionnement (plus faible que la barrière d'énergie de l'interface $\mathrm{Si}-\mathrm{SiO}_{2}$ ), il était suggéré que les porteurs ne pourraient atteindre des énergies suffisantes pour être injectés dans l'oxyde et dégrader les dispositifs. Cela est actuellement remis en question, des courants de grille ayant été observés dans des MOSFET de $0,14 \mu \mathrm{m}$ pour des tensions de drain aussi faibles que $2,35 \mathrm{~V}$ [4].

La localisation des injections et des défauts dans un MOSFET complique 1'interprétation des résultats. Toutefois, le système de base, i.e. l'hétérojonction $\mathrm{Si}_{-} \mathrm{SiO}_{2}$ étant identique dans les deux cas, on peut espérer que les mécanismes physiques de base (au niveau microscopique) restent les mêmes que lors de dégradations homogènes de capacités MOS [5]. Les différences entre les deux composants : l'absence de champ latéral dans les capacités MOS, le processus technologique plus complet des MOSFET, par exemple, peuvent conduire à des difficultés pour une comparaison quantitative des phénomènes. Toutefois, cela n'est probablement pas fondamental. Les défauts d'interface peuvent être, dans les deux cas, générés par injections d'électrons ou de trous. Plutôt qu'orthogonal, les injections de porteurs chauds dans les MOSFET submicroniques et les expériences d'injections homogènes sont complémentaires. Elles permettent.d'analyser différents aspects d'un même problème: la fiabilité des composants silicium VLSI.

\section{Etat de l'art.}

Différents auteurs ont proposé des modèles de dégradation des MOSFET basés sur les résultats des injections homogènes dans les capacités MOS. Sabnis et al. [6] et Hofmann et al. $[7,8]$ proposent des modèles basés sur les processus de capture d'électrons par des trous piégés pour la génération des états d'interface $[9,10]$. Cela correspond au fait que de nombreux travaux montrent que la création des états d'interface est maximale dans un MOSFET lorsque des électrons et des trous sont injectés simultanément à partir du canal (soit pour les conditions de fonctionnement entre $V_{\mathrm{g}}=V_{\mathrm{d}} / 3$ et $V_{\mathrm{g}}=V_{\mathrm{d}} / 2, V_{\mathrm{g}}$ et $V_{\mathrm{d}}$ étant respectivement la tension de grille et de drain) [11-17]. A plus faible $V_{\mathrm{g}}$, la dégradation est dominée par le piégeage de trous par des défauts d'oxyde pré-existants de type donneur [11]. Toutefois, d'autres auteurs font état d'une dégradation importante à $V_{\mathrm{g}} \approx V_{\mathrm{d}}$ [18-24]. Ces dégradations seraient dues au piégeage d'électrons dans l'oxyde par des défauts de type accepteur (soit pré-existants, soit crés au cours des injections) [18]. Ces défauts sont difficiles à mettre en évidence directement dans la mesure où ils sont probablement situés au-dessus du 
drain (zone de recouvrement grille-drain), donc dans une région qui n'est pas accessible par les mesures standards de caractéristiques courant-tension $(I-V)$ et/ou par la technique pompage de charge. Cette localisation est due au fait que l'injection d'électrons (maximale pour $V_{\mathrm{g}}=V_{\mathrm{d}}$ ) est située essentiellement au-dessus du drain, comme l'ont montré les simulations bi-dimensionnelles (2D) [15, 25-27]. Par ailleurs, d'autres simulations $2 \mathrm{D}$ prenant en compte la présence de défauts dans les MOSFET, ont mis en évidence que la création de charges négatives au-dessus du drain ou une augmentation de la densité d'états d'interface de type accepteur dans le canal donne exactement les mêmes effets sur la déformation des caractéristiques $I-V[28,29]$. Il est donc difficile, et hasardeux, de tirer des conclusions définitives uniquement à partir de l'étude de l'évolution des caractéristiques $I-V$ lors des expériences de vieillissement de MOSFET. De plus, ce type de dégradation, piégeage d'électrons à haut $V_{\mathrm{g}}$, semble plus important dans le cas de structure LDD (lightly doped drain) par comparaison aux structures conventionnelles [30-32]. Par ailleurs, la création de ces défauts d'oxyde de type accepteur a également été suggérée pour expliquer les expériences de vieillissement à bas $V_{\mathrm{g}}[19,33]$.

Il apparaît donc une certaine confusion et/ou complexité en ce qui concerne cette création de défauts accepteurs dans l'oxyde de grille, sa relation avec les conditions de polarisation appliquées sur le MOSFET et divers paramètres de technologie. Finalement, ces défauts accepteurs dans l'oxyde perturbent l'utilisation des techniques standards destinées à extraire les durées de vie des composants à partir de tests de vieillissement accélérés [33, 34]. De même, ils sont susceptibles de jouer un rôle important dans le cas de vieillissement en régime dynamique (impulsion sur la grille) pour lequel une dégradation plus importante est observée pour ce régime par rapport aux conditions de vieillissement statique [35-39]. Ceci a des répercutions directes dans le fonctionnement des circuits VLSI. Par exemple, il a été montré que les transistors fonctionnant avec un rapport cyclique élevé se dégradent plus vite que dans le cas opposé [18].

Finalement, notons qu'il n'y a quasiment aucun résultat sur la nature microscopique des défauts induits par les injections localisées dans les MOSFET submicroniques. Ceci est dô à la difficulté d'utiliser les techniques usuelles de caractérisation de défauts telles que la technique DLTS (Deep Level Transient Spectroscopy) et surtout la technique de Résonance Paramagnétique Electronique) (RPE) sur des composants de très faibles dimensions. Toutefois, une première approche en ce sens a été récemment proposée en utilisant une détection de la résonance paramagnétique sur les courants de génération-recombinaison des jonctions drain (ou source)-substrat du MOSFET (technique dite SDR (Spin Dependent Recombination) ou EDMR (Electrically Detected Magnetic Resonance)) [40-42]. Le sujet reste donc ouvert et prometteur pour de futurs travaux.

C'est cet aspect, création de défauts accepteurs dans l'oxyde de la zone de recouvrement grille-drain, que nous avons principalement étudié. Nous avons pour cela étendu le champ d'application de la technique de pompage de charge en montrant dans quelles conditions elle permet de détecter des charges négatives localisées au-dessus du drain [43]. Toutefois, cette technique étant limitée (voir Sect. 4), nous avons utilisé une nouvelle technique qui est fondée sur l'analyse de l'évolution du courant de grille des MOSFET au cours des expériences de vieillissement [44]. Ce courant de grille très faible (typiquement entre $10^{-17}$ et $10^{-12} \mathrm{~A}$ ) est mesuré par la technique de grille-flottante (GF). Enfin, certaines caractéristiques de ces défauts dans l'oxyde ont été obtenues par des mesures optiques de spectroscopie de photodépopulation, associées à des mesures d'émission assistée par champ électrique et à des mesures de section de capture. On a ainsi pu déterminer le diagramme de configuration de ces défauts et proposer une identification possible de leur nature [45-48]. A l'aide de ces techniques, nous avons analysé les conditions de création de ces défauts accepteurs dans des 
structures n-MOS de type LDD [49], La méthode de grille-flottante permet également de distinguer la création d'états d'interface et de charges négatives dans l'oxyde [47, 50], ceci en relation avec les conditions de vieillissement. La détermination des sections de capture de ces défauts a également été obtenue par cette technique de grille-flottante. Nous avons ainsi pu démontrer la similitude de nature des défauts crés par injection d'électrons lors de vieillissement à haut $V_{\mathrm{g}}$ et injection de trous à bas $V_{\mathrm{g}}$ [48]. Ceci a permis une étude exhaustive des défauts induits dans les MOSFET [49]. Elle a été couplée à des simulations bidimensionnelles de dispositifs avec le logiciel IMPACT [51, 52] dans lequel les défauts (états d'interface, défauts dans l'oxyde de grille) précédemment caractérisés sont pris en compte $[53,54]$. On a étudié leurs effets sur les caractéristiques $I-V$ et par comparaison avec l'expérience, on réalise ainsi une étude auto-cohérente du vieillissement des MOSFET submicroniques [49].

\section{Technique de la grille-flottante.}

L'injection de porteurs chauds à partir du canal donne naissance à un courant de grille très faible dans les n-MOSFET, courant d'électrons pour $V_{\mathrm{g}}>V_{\mathrm{d}} / 2$ et courant de trous pour $V_{\mathrm{g}}<V_{\mathrm{d}} / 2[8,55]$. Ces courants, de 1'ordre de $10^{-17}-10^{-12} \mathrm{~A}$, sont mesurables par la technique de la grille-flottante (GF) [55-57]. Ce courant de grille est très sensible aux phénomènes de création de défauts et de piégeage de charges dans l'oxyde [44]. Comme l'injection des porteurs donnant naissance à ce courant de grille est très localisée, la mesure du courant de grille est donc une sonde locale pour analyser les dommages induits par les porteurs chauds. En particulier, nous avons ainsi démontré la création de défauts de type accepteur dans l'oxyde de la zone de recouvrement grille-drain des MOSFET submicroniques [44, 47-49]. L'analyse précise des dommages dans cette zone est impossible par les techniques conventionnelles: mesure des dérives des caractéristiques $I-V$, tension de seuil $V_{1}$, transconductance $g_{\mathrm{m}}$, et dans une moindre mesure par la technique de pompage de charge. Cette technique GF est donc très performante et elle apporte des informations nouvelles permettant de confirmer les hypothèses déduites de mesures plus globales de type $I-V$.

3.1 PRINCIPE DE LA TECHNIQUE. - Le potentiel d'une grille isolée est modifié par les porteurs atteignant la grille (électron ou trou en fonction du champ électrique dans l'oxyde). La variation correspondante du courant de drain $\Delta I_{\mathrm{d}}$ pendant un intervalle de temps $\Delta t$ permet de mesurer indirectement des courants de grille très faibles par la relation $I_{\mathrm{g}}=C_{\mathrm{g}} \Delta I_{\mathrm{d}} / g_{\mathrm{m}} \Delta t$ où $C_{\mathrm{g}}$ est la capacité de grille (incluant la capacité du plot de contact). La tension de grille est directement donnée par $V_{\mathrm{g}}-V_{\mathrm{t}}=I_{\mathrm{d}} / g_{\mathrm{m}}$. La figure 1 donne un exemple typique des courants d'électrons et de trous mesurés sur un MOSFET non vieilli. Le choix de la tension de drain pendant la mesure du courant de grille est très critique. Une tension trop importante est susceptible de dégrader très rapidement le composant ce qui perturberait la mesure. Une tension trop faible ne permet pas une injection suffisante de porteurs dans l'oxyde, conduisant à des temps de mesure prohibitifs pour détecter un changement significatif de $I_{\mathrm{d}}$. Une étape de calibration est donc nécessaire avant toute mesure. Pour vérifier l'absence de self-dégradation pendant la mesure, plusieurs mesures sont effectuées l'une après l'autre et l'on ne doit pas observer de modification significative du courant de grille. De même, les mesures de pompage de charge faites avant et après la mesure de GF doivent être identiques.

Nous avons appliqué cette technique GF à la caractérisation des défauts induits par injections de porteurs chauds. La figure 2 donne un exemple de l'évolution du courant de grille d'électrons lors d'un vieillissement par injection d'électrons à $V_{\mathrm{g}}=V_{\mathrm{d}}=8,5 \mathrm{~V}$ pendant $4 \times 10^{4} \mathrm{~s}[44,49]$. Le MOSFET est de type LDD de géométrie (dessinée) $1 \mu \mathrm{m} \times 100 \mu \mathrm{m}$ 


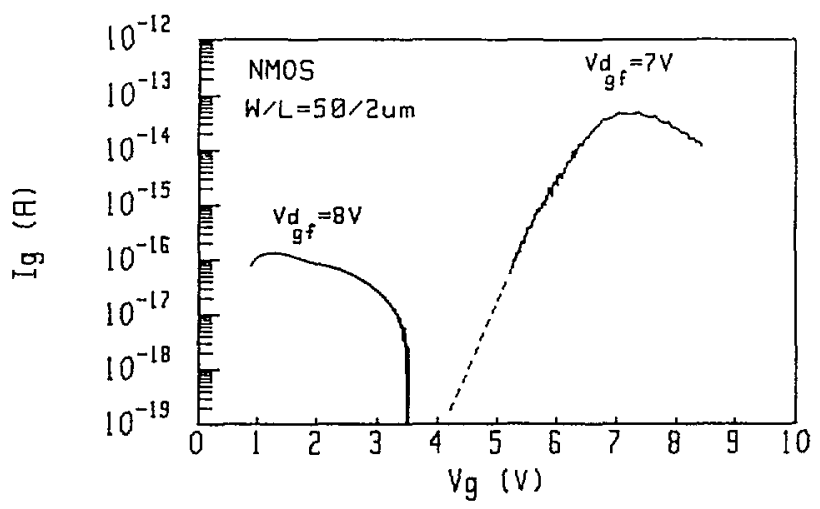

Fig. 1. - Courants de grille d'électrons $\left(V_{\mathrm{g}}>5 \mathrm{~V}\right)$ et de trous $\left(V_{\mathrm{g}}<4 \mathrm{~V}\right)$ mesurés par la technique de grille-flottante sur un n-MOSFET (d'après Réf. [47]).

[Electron $\left(V_{\mathrm{g}}>5 \mathrm{~V}\right)$ and hole $\left(V_{\mathrm{g}}<4 \mathrm{~V}\right)$ gate currents measured by the floating-gate technique (from Ref. [47]).]

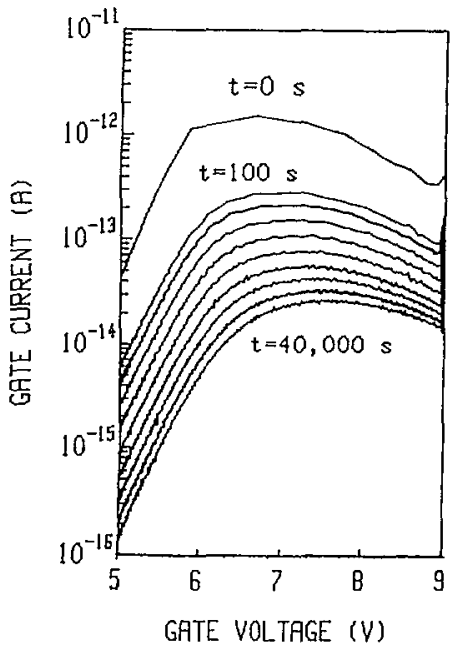

a)

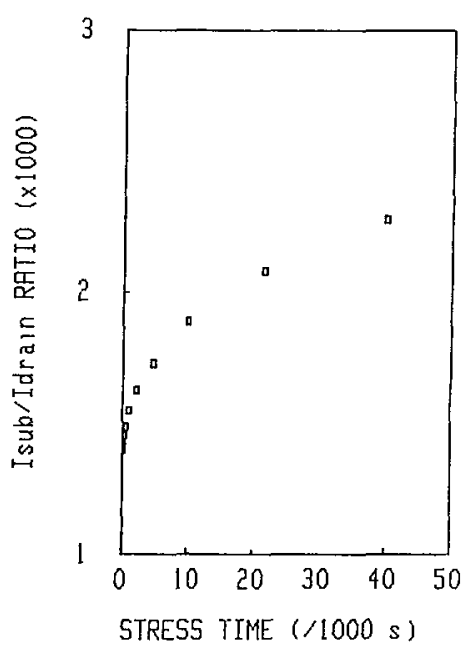

b)

Fig. 2. - (a) Evolutions du courant de grille électronique mesurées par grille-flottante (à $V_{\mathrm{d}}=6 \mathrm{~V}$ ) en fonction du temps de vieillissement $(0,100,215,464,1000,2154,4640,10000,21544$ et $40000 \mathrm{~s})$ à $V_{g}=V_{d}=8,5 \mathrm{~V}$. (b) Evolution correspondante du rapport courant de substrat sur courant de drain (d'après Réf. [44]).

[(a) Electronic gate current evolutions measured by floating-gate technique (at $V_{\mathrm{d}}=6 \mathrm{~V}$ ) after different aging times $\left(0,100,215,464,1000,2154,4640,10000,21544\right.$ and 40000 s) at $V_{\mathrm{g}}=V_{\mathrm{d}}=8.5 \mathrm{~V}$. (b) Corresponding evolutions of the substrate to drain current ratio (from Ref. [44]).]

$\left(L_{\text {eff }}=0,85 \mu \mathrm{m}\right)$ et d'épaisseur d'oxyde $200 \AA$. On observe une décroissance importante de $l_{\mathrm{g}}$ (à tout $V_{\mathrm{g}}$ ) sur plus de 2 décades selon une loi en puissance du temps. Selon le modèle de l'électron chanceux [58], les deux paramètres qui gouvernent le courant de grille sont : le maximum du champ électrique latéral $\left(E_{\mathrm{m}}\right)$ et la hauteur effective de la barrière de potentiel à 
l'interface $\mathrm{Si}_{-\mathrm{SiO}_{2}}\left(\Phi_{\mathrm{b}}\right)$. L'évolution de $E_{\mathrm{m}}$ est mesurée par le rapport $I_{\text {sub }} / I_{\mathrm{d}}$ (Fig. 2). L'augmentation observée devrait conduire à une augmentation de $I_{\mathrm{g}}$ en opposition avec les résultats expérimentaux de la figure 2. Nous en concluons que la diminution de $I_{\mathrm{g}}$ est ici dominée par une augmentation de $\Phi_{\mathrm{b}}$. Ces deux augmentations sont dues à la création de charges négatives dans l'oxyde. Une évaluation quantitative de $\Delta \Phi_{\mathrm{b}}$ en utilisant le formalisme de l'électron chanceux est trop grossière pour en extraire précisément une concentration de charge à l'interface. Toutefois une première estimation montre que $10^{12}$ charge $/ \mathrm{cm}^{2}$ sur $50 \AA$ à partir de l'interface peuvent induire une variation de $I_{\mathrm{g}}$ sur 3 décades. Une analyse plus fine des évolutions de $I_{\mathrm{g}}$ nécessiterait des simulations $2 \mathrm{D}$ du courant de grille prenant en compte les distributions des charges dans l'oxyde. L'importante modification de $I_{\mathrm{g}}$ (Fig. 2) pour un vieillissement à $V_{\mathrm{g}}=V_{\mathrm{d}}$ ne s'accompagne pas d'une modification significative de $V_{\mathrm{t}}$, ni d'un décalage global des flancs de la caractéristique de pompage de charge $I_{\mathrm{cp}}-V_{\mathrm{b}}$ vers les tensions plus positives comme cela est observé lors de la création de charges négatives audessus du canal [59]. Cela indique que les défauts accepteurs sont localisés au-dessus du drain dans une zone inaccessible par ces techniques. Seule une faible modification de la caractéristique $I_{\mathrm{cp}}-V_{\mathrm{b}}$ permet de détecter ces charges négatives [43] (voir Sect. 4). De même, une mesure de GF en mode inverse (source et drain inversés) permet de démontrer la localisation du côté du drain et l'absence de dommage à la source [47, 49].

3.2 PROPRIÉtÉs DE CAPTURE DES DÉFAuTS D'OXYDe ACCEPTEURS. - Après le vieillissement de longue durée $\left(4 \times 10^{4} \mathrm{~s}\right)$ responsable de la création de défauts dans l'oxyde, on réalise de brèves $(100 \mathrm{~s})$ injections d'électrons à $V_{\mathrm{g}}=V_{\mathrm{d}}$ ou de trous à $V_{\mathrm{g}}=V_{\mathrm{d}} / 4$ pour induire des changements d'états de charge de ces défauts par la capture des électrons ou des trous injectés dans l'oxyde. Ces changements d'état de charge sont également observés par l'évolution temporelle du courant de grille mesurée par GF [47, 48]. Dans le cas des MOSFET LDD, nous avons comparé les propriétés de capture des défauts crés par vieillissement à haut $V_{\mathrm{g}}\left(V_{\mathrm{g}}=V_{\mathrm{d}}\right)$ et bas $V_{\mathrm{g}}\left(V_{\mathrm{g}}=V_{\mathrm{d}} / 4\right)$. Les cinétiques d'évolution du maximum de $I_{\mathrm{g}}$ sont données à la figure 3 dans le cas d'un vieillissement à $V_{\mathrm{g}}=V_{\mathrm{d}}$. Sous l'effet d'une injection d'électrons, $I_{\mathrm{g}}$ diminue ce qui correspond à une transition $0 \rightarrow-$ (capture d'un électron) et la dépendance temporelle de $I_{\mathrm{g}}$ est correctement ajustée par une cinétique d'ordre 1 avec une constante de temps de capture $\tau_{\mathrm{e}}=55,4 \mathrm{~ms}$. On en déduit une section de capture pour les électrons $\sigma_{\mathrm{e}}=e / J_{\mathrm{inj}} \tau_{\mathrm{e}}=1,4 \times 10^{-14} \mathrm{~cm}^{2}$ où $J_{\mathrm{inj}}$ est la densité de courant injectée. Sous l'effet de l'injection de trous, $I_{\mathrm{g}}$ augmente ce qui correspond à la transition inverse $\rightarrow 0$, mais la loi est plus compliquée dans ce cas. Elle est correctement ajustée par deux cinétiques d'ordre 1 avec des constantes de temps $\tau_{\mathrm{h} 1}=91 \mathrm{~ms}$ et $\tau_{\mathrm{h} 2}=1,96 \mathrm{~s}$. Cependant, comme le courant de trous injectés est très faible $\left(\sim 10^{-16} \mathrm{~A}\right.$ mesuré par GF) cela conduit à des sections de capture géantes non réaliste physiquement $\left(>10^{-10} \mathrm{~cm}^{2}\right)$. Nous pensons que dans ce cas, la capture de trous n'est pas le mécanisme unique qui a lieu. Nous pouvons raisonnablement supposer qu'il existe un depiégeage non négligeable des électrons sous l'effet du champ électrique (de l'ordre de quelques $\mathrm{MV} / \mathrm{cm}$ ).

Pour le vieillissement à $V_{\mathrm{g}}=V_{\mathrm{d}} / 4$, les mesures de cinétiques par GF donnent des résultats très similaires : $\tau_{\mathrm{e}}=58,3 \mathrm{~ms}$ et $\sigma_{\mathrm{e}}=1,37 \times 10^{-14} \mathrm{~cm}^{2}$ pour la transition $0 \rightarrow-$, $\tau_{\mathrm{h} 1}=32,4 \mathrm{~ms}$ et $\tau_{\mathrm{h} 2}=1,72 \mathrm{~s}$ pour la transition $\rightarrow 0$ [48]. Cela indique que les mêmes défauts sont créés dans l'oxyde lors de l'injection d'électrons et de trous chauds au cours des expériences de vieillissement de nos dispositifs LDD. Notons cependant que ces valeurs de sections de capture sont assez approximatives. En effet, il n'a pas été tenu compte de : i) la modification de $J_{\text {inj }}$ au cours des phases d'injections due à la modification des champs électriques lors du changement d'état de charge des défauts, ii) la valeur de $J_{\text {inj }}$ dépend de la surface effective de la zone d'injection et de la zone dégradée qui ne sont pas accessibles à l'expérience. On utilise un ordre de grandeur donné par les simulations $2 \mathrm{D}(\sim 0,1 \mu \mathrm{m})$. 


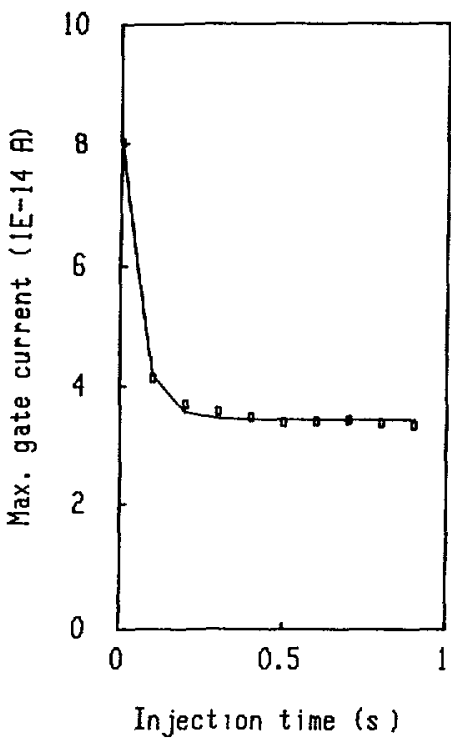

a)

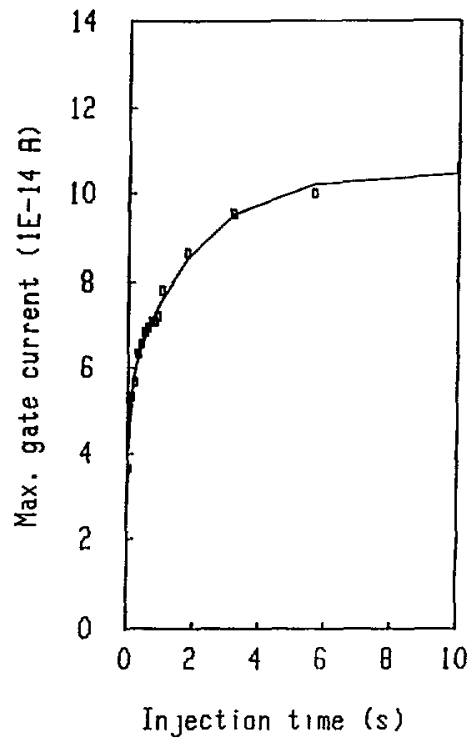

b)

Fig. 3. - Cinétique d'évolution du maximum du courant de grille (a) durant les courtes injections d'électrons (CIE) et (b) durant les courtes injections de trous (CIT) appliquées après un vieillissement à $V_{\mathrm{g}}=V_{\mathrm{d}}=8,5 \mathrm{~V}$. Les carrés représentent les mesures, les lignes représentent les ajustements théoriques (d'après Réfs. [47, 48]).

[Time kinetics of the maximum gate current (a) during the short electron injections (SEI) and (b) the short hole injections (SHI) applied after a long time aging at $V_{\mathrm{g}}=V_{\mathrm{d}}=8.5 \mathrm{~V}$. Squares are the measured data and lines are the fitted kinetics (from Refs. [47, 48]).]

Nos résultats sont à comparer avec ceux de Doyle et al. qui ont rapporté des valeurs différentes $[33,60]$. Ils font état d'une section de capture $\sigma_{\mathrm{e}} \approx 3 \times 10^{-18} \mathrm{~cm}^{2}$ pour les défauts créśs à $V_{\mathrm{g}}=V_{\mathrm{d}}$ et de deux sections de capture $\sigma_{e 1} \approx 3 \times 10^{-14} \mathrm{~cm}^{2}$ et $\sigma_{\mathrm{e} 2} \approx 2 \times 10^{-15} \mathrm{~cm}^{2}$ pour les défauts créés à $V_{\mathrm{g}}=V_{\mathrm{d}} / 4$. Les approximations citées ci-dessus concernant la précision des mesures étant valables dans les 2 cas, ces résultats ne sont pas forcément contradictoires. En effet, notons que les résultats de Doyle et al. à haut et bas $V_{\mathrm{g}}$ ont été obtenus pour des technologies de provenance différentes et non pas sur les mêmes dispositifs comme dans notre étude.

Une section de capture $\sigma_{\mathrm{e}} \approx 1,4 \times 10^{-14} \mathrm{~cm}^{2}$ peut sembler surprenante pour la capture d'un électron par un centre neutre si on se réfère à la classification standard [61]. Toutefois, celle-ci n'est pas rigoureusement valable. Il a été montré très clairement que des défauts accepteurs peuvent posséder une section de capture importante (de l'ordre de $10^{-13} \mathrm{~cm}^{2}$ ) à condition de présenter une relaxation de réseau importante lors d'un changement d'état de charge [62]. Une large relaxation de réseau a été expérimentalement vérifiée pour les défauts d'oxyde crés par injection de trous à $V_{\mathrm{g}}=V_{\mathrm{d}} / 4$, par des expériences de photodépopulation couplées à des expériences d'émission assistée par champ électrique [45, 46] (voir Sect. 5). Nous pensons donc que la section de capture de $1,4 \times 10^{-14} \mathrm{~cm}^{2}$ mesurée lors de nos expériences utilisant la méthode GF est parfaitement réaliste et qu'elle est associée à des défauts accepteurs dans l'oxyde. 
3.3 ETATS D'INTERFACE ET/OU DÉFAUTS DANS L'OXYDE. - En collaboration avec Doyle et al., nous avons montré que la technique GF est capable de discerner la création d'états d'interface de celle de charges dans l'oxyde [47, 50]. En effet, les charges stockées sur des états d'interface peuvent également induire des modifications de $I_{\mathrm{g}}$. Dû au fait que l'état de charge des défauts à l'interface dépend de la position du niveau de Fermi à cette interface, les modifications de la caractéristique $I_{\mathrm{g}}-V_{\mathrm{g}}$ ne seront pas uniformes. La caractéristique $I_{\mathrm{g}}-V_{\mathrm{g}}$ (cas n-MOSFET) sera modifiée en régime linéaire $\left(V_{\mathrm{g}}>V_{\mathrm{d}}\right)$ dans le cas d'états d'interface accepteurs (chargés négativement pour le niveau de Fermi près de la bande de conduction), et en régime de saturation $\left(V_{\mathrm{g}}<V_{\mathrm{d}}\right)$ pour des états d'interface donneurs (chargés positivement si le substrat sous le canal est suffisamment déplété). La mise en évidence du premier cas (états d'interface accepteurs) a été obtenue sur des n-MOSFET nonLDD $(2 \mu \mathrm{m} \times 50 \mu \mathrm{m}$, épaisseur d'oxyde $400 \AA)$ stressés à $V_{\mathrm{g}}=V_{\mathrm{d}} / 2\left(V_{\mathrm{d}}=7 \mathrm{~V}\right.$ durant $\left.4 \times 10^{4} \mathrm{~s}\right)$. Dans le cas de MOSFET non-LDD, il a été montré que la création d'états d'interface est le mécanisme dominant aux conditions $V_{\mathrm{g}}=V_{\mathrm{d}} / 4$ [11-17], alors que la création de charges négatives dans l'oxyde (défauts accepteurs) est la principale cause de dégradation à $V_{\mathrm{g}}=V_{\mathrm{d}}$ [18-24]. La figure 4 montre l'évolution des caractéristiques $I_{\mathrm{g}}-V_{\mathrm{g}}$ mesurées par GF pour les deux conditions de vieillissement. Les caractéristiques figure $4 a$ sont révélatrices de la création d'états d'interface accepteurs près du drain avec une distribution énergétique principalement localisée près de la bande de conduction [47, 50]. Dans la région de saturation $\left(V_{\mathrm{g}}<V_{\mathrm{d}}\right)$, le niveau de Fermi (près du drain dans la région de pincement) est loin de la

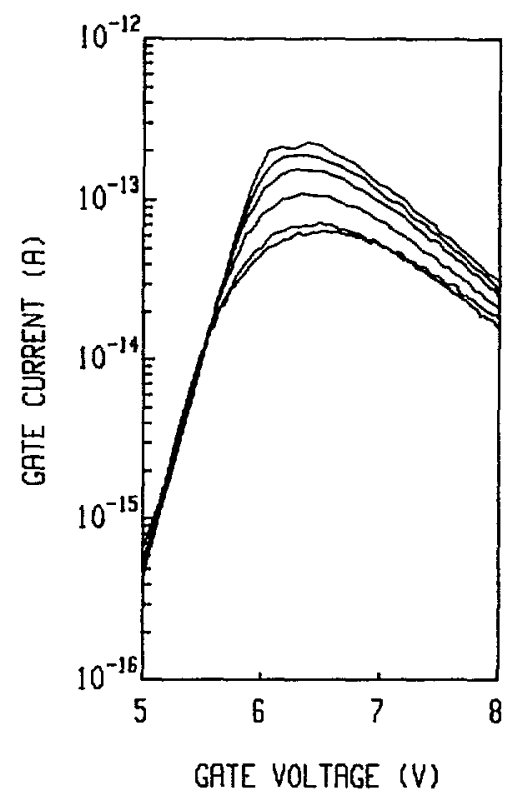

a)

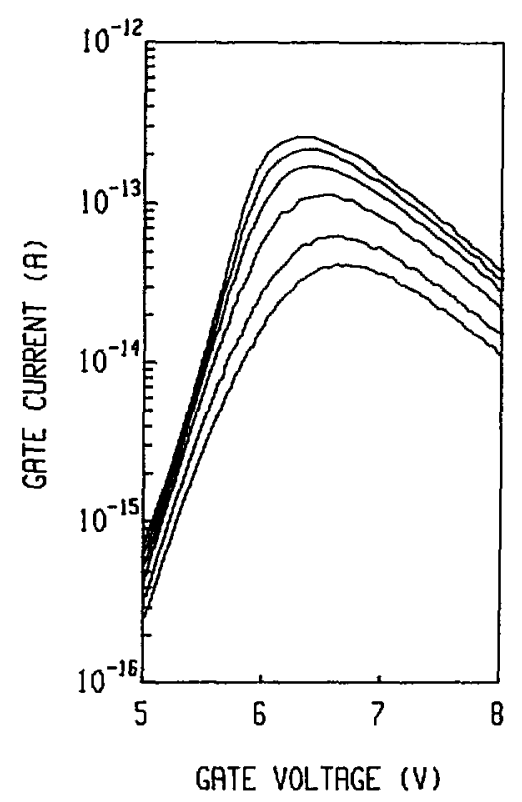

b)

Fig. 4. - Evolution des caractéristiques $I_{\mathrm{g}}-V_{\mathrm{g}}$ lors de la création (a) d'états d'interface (vieillissement à $V_{\mathrm{g}}=V_{\mathrm{d}} / 2$ ) et (b) de charges dans l'oxyde (vieillissement à $V_{\mathrm{g}}=V_{\mathrm{d}}$ ). Temps de vieillissement : 0,10 , $100,1000,10000$ et $40000 \mathrm{~s}$ (d'après Réfs. [47, 50]).

[Evolution of the $I_{\mathrm{g}}-V_{\mathrm{g}}$ characteristics for the creation of (a) interface states (aging at $V_{\mathrm{g}}=V_{\mathrm{d}} / 2$ ) and (b) oxide charges (aging at $V_{\mathrm{g}}=V_{\mathrm{d}}$ ). Aging times : $0,10,100,1000,10000$ and $40000 \mathrm{~s}$ (from Refs. $[47,50])$.] 
bande de conduction (au moins à $0,15 \mathrm{eV}$ [50]) et les états d'interface sont neutres. Ils ne présentent pas une barrière à l'injection des électrons dans l'oxyde et en conséquence, il n'y a aucun changement de la caractéristique $I_{\mathrm{g}}-V_{\mathrm{g}}$. En régime linéaire, le niveau de Fermi est très proche de la bande de conduction et les états d'interface sont chargés négativement, ce qui induit la décroissance de $I_{\mathrm{g}}$ observé sur la fígure 4a. Dans le cas de charges négatives dans l'oxyde (vieillissement à $V_{\mathrm{g}}=V_{\mathrm{d}}$, Fig. 4b), l'état de charge ne dépend pas du niveau de Fermi, et on observe une décroissance de $I_{\mathrm{g}}$ sur l'ensemble de la caractéristique de GF. Au contraire, les défauts d'oxyde sont sensibles aux injections post-vieillissement, comme nous l'avons vu au paragraphe 3.2, alors que ces injections n'ont aucune influence sur l'état de charge des états d'interface qui ne dépend que du niveau de Fermi pendant la mesure de GF. La figure 5 illustre clairement cette différence de comportement, où l'on observe aucun effet des courtes injections d'électrons et de trous dans le cas d'une création d'états d'interface. Ces propriétés différentes permettent donc également de distinguer les deux types de défauts [47, 50].

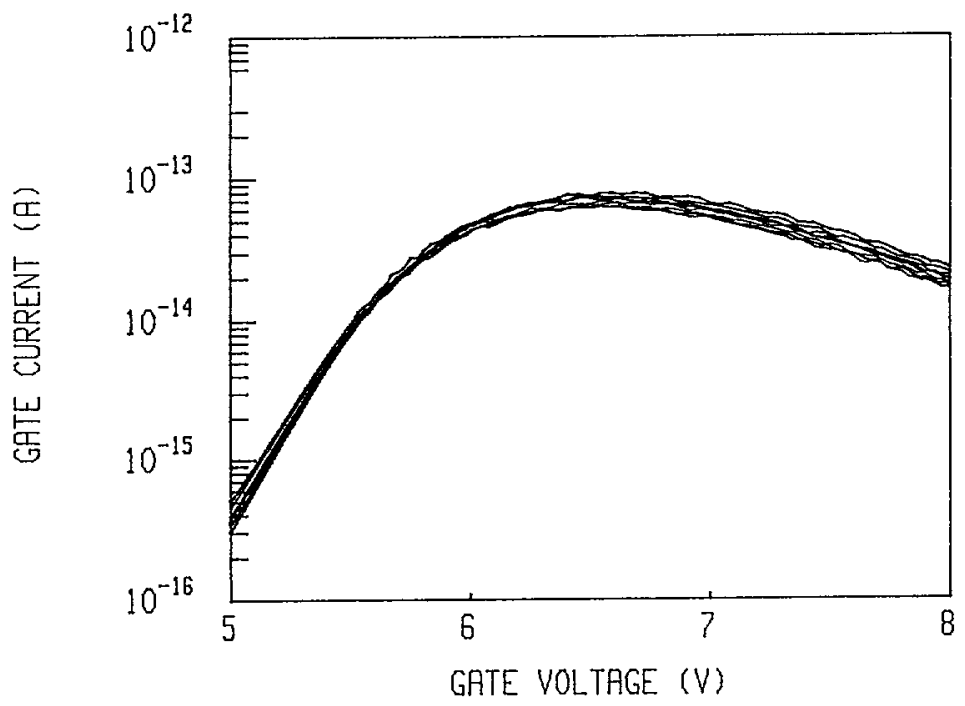

Fig. 5. - Caractéristique $I_{\mathrm{g}}-V_{\mathrm{g}}$ mesurée par grille-flottante pour quatre cycles CIE et CIT appliqués sur le MOSFET après la création d'états d'interface uniquement (d'après Réf. [47]).

$\left[I_{\mathrm{g}}-V_{\mathrm{g}}\right.$ characteristics measured by the floating-gate technique for 4 cycles of SEI and SHI phases applied on the MOSFET after the creation of interface states only (from Ref. [47]).]

\section{La technique de pompage de charge.}

La technique de pompage de charge permet facilement la caractérisation des états d'interface et des charges d'oxyde localisés au-dessus du canal d'un MOSFET submicronique [59, 63, 64]. Nous avons montré comment les charges négatives localisées au-dessus du drain dans la zone de recouvrement grille-drain pouvaient être détectées par cette technique [43]. Dans le cas d'un n-MOSFET, le flanc de la caractéristique $I_{\mathrm{cp}}-V_{\mathrm{b}}$ présente une queue de courant pour les tensions $V_{\mathrm{b}}$ les plus négatives (Figs. 6 et 7). Heremans et al. ont démontré que cette queue de courant provient de la variation locale de la tension de bande plate et de la tension de seuil 


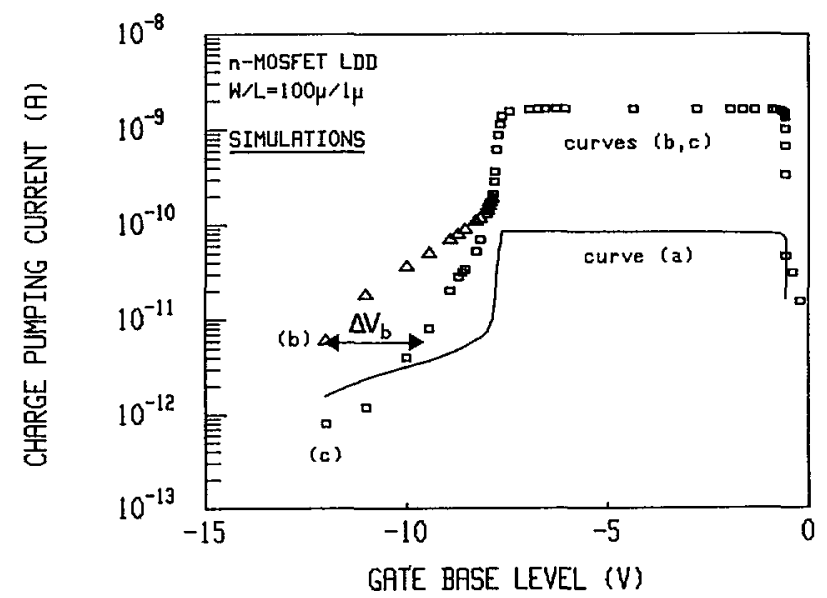

Fig. 6. - Simulations des caractéristiques de pompage de charge : (a) MOSFET non-vieilli, (b) après création d'états d'interface, (c) après création d'états d'interface et de charges négatives dans l'oxyde audessus du drain (d'après Réf. [43]).

[Simulated charge pumping characteristics : (a) virgin MOSFET, (b) after interface state creation, (c) after creation of interface states and negative oxide charges above the drain (from Ref. [43]).]

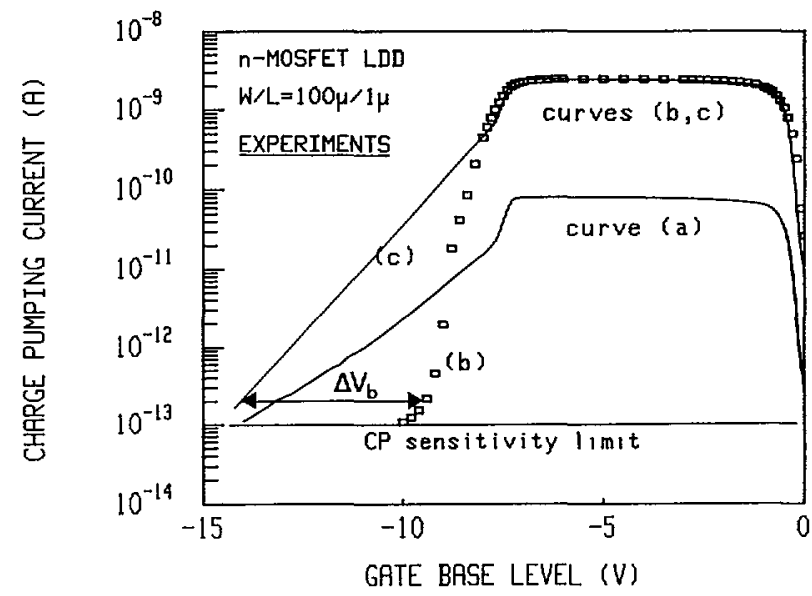

Fig. 7. - Courbes de pompage de charge mesurées : (a) MOSFET non-vieilli, (b) après vieillissement et $\mathrm{CIE}$. La courbe $c$ est une courbe calculée en ne prenant en compte que la création des états d'interface (d'après Réf. [43]).

[Measured charge pumping curves : (a) virgin MOSFET, (b) after aging and SEI. Curve $c$ is calculated taking into account the interface state creation alone (from Ref. [43]).]

près du drain à cause de la variation du dopage dans cette région du transistor [59]. Pour les tensions $V_{\mathrm{b}}$ les plus fortement négatives, ces auteurs mettent en évidence que le courant $I_{\mathrm{cp}}$ est caractéristique des défauts près du drain et qu'une certaine pénétration au-dessus du drain est possible pour les tensions extrêmes ( $\sim 0,05 \mu \mathrm{m}$ dans leur cas). Reprenant cette approche, nous avons simulé la caractéristique $I_{\mathrm{cp}}-V_{\mathrm{b}}$ en prenant en compte un paquet de 
charges négatives au-dessus du drain. Les profils de dopage le long de l'interface ont été simulés par le logiciel IMPACT pour un dispositif non-LDD, et on a introduit une distribution rectangulaire de charges négatives induisant un décalage local des tensions $V_{\mathrm{fb}}$ et $V_{\mathrm{t}}$. Le résultat des simulations montre une dérive vers les tensions plus positives de la queue de la caractéristique $I_{\mathrm{cp}}-V_{\mathrm{b}}$, le reste de la courbe restant inchangé (Fig. 6). Ceci diffère donc notablement de l'effet de charges au-dessus du canal qui décalent nettement les deux flancs de la caractéristique $I_{\mathrm{cp}}-V_{\mathrm{b}}$ [59]. Le décalage $\Delta V_{\mathrm{b}}$ est, au premier ordre, en accord avec la densité de charge $N_{\text {ot }}$ par la relation classique $\Delta V_{\mathrm{b}}=q N_{\text {ot }} / C_{\text {ox }}$. L'augmentation de $I_{\mathrm{cp}}$ représentée par la courbe (b) figure 6 correspond uniquement à une création d'états d'interface selon le profil triangulaire près du drain. Le maximum de $D_{\text {it }}$ a été ajusté par rapport aux résultats expérimentaux. Prendre un profil plus réaliste, extrait des mesures de pompage de charge [65], ne modifie pas les conclusions obtenues.

Le comportement prédit par les simulations a été observé expérimentalement sur des nMOSFET LDD vieillis à $V_{\mathrm{g}}=V_{\mathrm{d}} / 4\left(V_{\mathrm{d}}=8,5 \mathrm{~V}\right)$ pendant $4 \times 10^{4} \mathrm{~s}$ (même dispositif que pour les mesures de GF, Sect. 3) [43, 49]. La figure 7, courbe (b), montre la caractéristique $I_{\mathrm{cp}}-V_{\mathrm{b}}$ après l'application d'une courte injection d'électrons (CIE) à $V_{\mathrm{g}}=V_{\mathrm{d}}$ destinée à charger négativement les défauts accepteurs créés par le vieillissement. Ce comportement est parfaitement conforme aux simulations, et prouve l'existence de charges négatives localisées au-dessus de drain, en accord avec les résultats des mesures de GF [44, 49] (voir Sect. 3). Le fait que la courbe (b) croise la courbe (a) correspondant au dispositif non vieilli ne peut s'expliquer que par une forte densité de charges négatives, ici $N_{\text {ot }} \approx 3,7 \times 10^{12} \mathrm{~cm}^{-2}$ Le même comportement a été observé pour d'autres conditions de vieillissement mais avec des amplitudes plus faibles (voir Sect. 6). Il est donc intéressant de coupler les mesures de GF et de pompage de charge pour avoir une caractérisation la plus complète possible de ces défauts accepteurs dans l'oxyde de la zone de recouvrement entre grille et drain des MOSFET.

\section{Propriétés optiques des défauts d'oxyde.}

Pour avoir plus d'informations sur les défauts d'oxyde crés par l'injection de porteurs chauds, nous avons combiné une étude des propriétés optiques et électriques de ces défauts $[45,46]$. Dans un premier temps, les défauts-crés (pour cette étude à $V_{\mathrm{g}}=V_{\mathrm{d}} / 4=2 \mathrm{~V}$ pendant $6 \times 10^{4} \mathrm{~s}$ dans un MOSFET non-LDD de $2 \mu \mathrm{m} \times 50 \mu \mathrm{m}, t_{\mathrm{ox}}=400 \AA$ ) sont remplis par des électrons au cours d'une courte injection de $100 \mathrm{~s}$ à $V_{\mathrm{g}}=V_{\mathrm{d}}$. Les effets de changement d'états de charge des défauts sont observés par la mesure des caractéristiques $I_{\mathrm{d}}-V_{\mathrm{g}}$ en régime linéaire. On analyse ensuite l'émission de ces électrons, soit sous l'influence de photons d'énergie entre 1 et $4 \mathrm{eV}$ (spectroscopie de photodépopulation) ou par l'application d'un champ électrique de l'ordre de 2,5 à 3,5 MV/cm dans l'oxyde (émission assistée par champ électrique). Pour les mesures de photodépopulation, le MOSFET est polarisé avec une faible tension entre grille et drain de manière à collecter les électrons photoémis par le drain. Le champ dans l'oxyde est inférieur à $1 \mathrm{MV} / \mathrm{cm}$ pour éviter les effets de dépiégeage dus au champ. On utilise un monochromateur, et le faisceau incident arrive sur le MOSFET avec un angle de $\sim 30^{\circ}$. En effet, les rayonnements visibles et UV ne pouvant pénétrer par la grille poly-Si, le faisceau pénètre dans l'oxyde de grille par le côté du drain pour avoir un effet marqué sur les défauts dans la zone de recouvrement grille-drain. On limite l'énergie des photons à $4 \mathrm{eV}$ pour éviter une photoinjection de trous à partir du substrat de type p. Pour les expériences d'émission assistée par champ, une tension de drain importante $(9-15 \mathrm{~V})$ est appliquée sur le MOSFET à l'état bloqué $\left(V_{\mathrm{g}}=0 \mathrm{~V}\right)$. Le champ électrique est ainsi localisé dans la région de recouvrement grille-drain, et seul les défauts de cette région seront observés lors de l'expérience. Dans les deux cas, on suit les cinétiques 


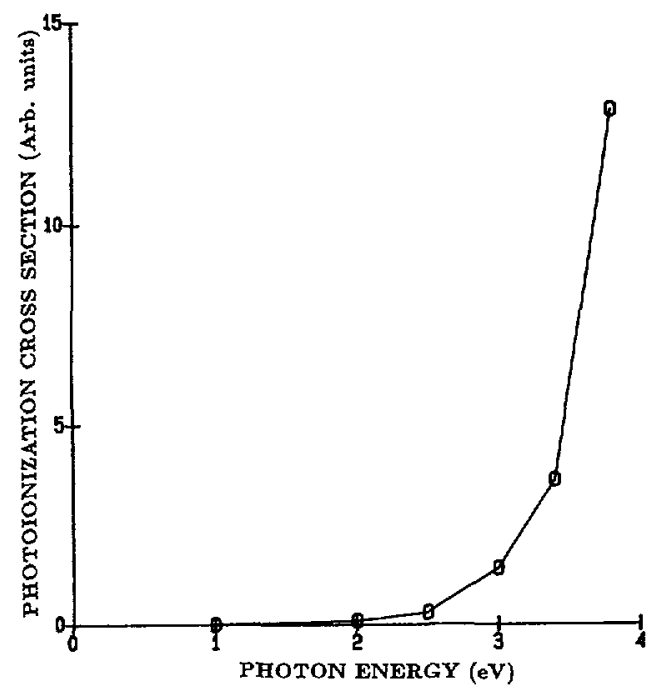

Fig. 8. - Section efficace de photoionisation des défauts d'oxyde induits par le vieillissement (d'après Réfs. $[45,46])$.

[Photoionization cross-section of the oxide defects induced by the aging (from Refs. [45, 46]).]

d'émission par le retour de la caractéristique $I_{\mathrm{d}}-V_{\mathrm{g}}$ vers celle mesurée avant le remplissage des défauts. Les cinétiques de photodépopulation suivent une loi du premier ordre qui permet d'extraire la section efficace de photoionisation $\sigma^{0}(h \nu)$. La figure 8 donne la courbe obtenue qui présente un seuil pour une énergie entre 2,5 et $3,5 \mathrm{eV}$. Nous retiendrons donc une énergie de photoionisation $E_{\mathrm{T}}^{0} \approx 3,0 \pm 0,5 \mathrm{eV}$ pour ces défauts d'oxyde. Pour les expériences d'émission assistée par le champ électrique, la figure 9 donne la dépendance du taux d'émission en fonction, soit du champ électrique $F$, soit de $\sqrt{F}$. Ce dernier cas, correspond à un effet Poole-Frenkel pour l'émission d'un électron par un défaut de type donneur [66-68], tandis que la dépendance en $F$ correspond à un défaut présentant un potentiel répulsif à courte distance, c'est-à-dire à un défaut de type accepteur [68-70]. Dans les deux cas, le terme pré-exponentiel à champ électrique nul est donné par $\tau_{\mathrm{e}}(0)=\left(\sigma_{\mathrm{e}} \gamma T^{2}\right)^{-1} \exp \left(E_{\mathrm{T}} / k T\right)$ où $E_{\mathrm{T}}$ est l'énergie d'ionisation thermique, $\sigma_{\mathrm{e}}$ la section de capture et $\gamma$ une constante dépendant du matériau. Selon, le type de dépendance en champ électrique que l'on considère, les valeurs de $\tau_{\mathrm{e}}(0)$ qui sont extraites des ajustements sur les points expérimentaux diffèrent. Pour des sections de capture comprises entre $10^{-19}$ et $10^{-14} \mathrm{~cm}^{2}$ (ce qui semble raisonnable pour les défauts dans $\mathrm{SiO}_{2}$ ) [61], on obtient alors les valeurs suivantes : $E_{\mathrm{T}} \approx 3,0 \pm 0,1 \mathrm{eV}$ si le défaut est donneur et $E_{\mathrm{T}} \approx 1,7 \pm 0,1 \mathrm{eV}$ si le défaut est accepteur [45, 46]. La déconvolution entre une dépendance en $F$ ou $\sqrt{F}$ demanderait une étude sur une gamme plus importante de champ électrique. Ceci n'a pas été possible à $300 \mathrm{~K}$, dû aux limites de constante de temps imposées par la méthode de mesure des cinétiques d'émission $\left(10^{-2}\right.$ à $\left.10^{6} \mathrm{~s}\right)$. Une étude en température pour moduler plus largement les constantes de temps d'émission est envisageable, mais peut se révéler plus difficile à interpréter si le processus d'émission est assisté par les phonons.

En utilisant la représentation des défauts dans le formalisme d'un diagramme de configuration, la différence entre l'énergie d'ionisation optique $E_{\mathrm{T}}^{0}$ et thermique $E_{\mathrm{T}}$ donne une idée de l'importance de la relaxation de réseau lors d'un changement d'état de charge [71]. Une différence importante donne un paramètre de Franck-Condon $d_{\mathrm{FC}}=E_{\mathrm{T}}^{0}-E_{\mathrm{T}}$ important, 


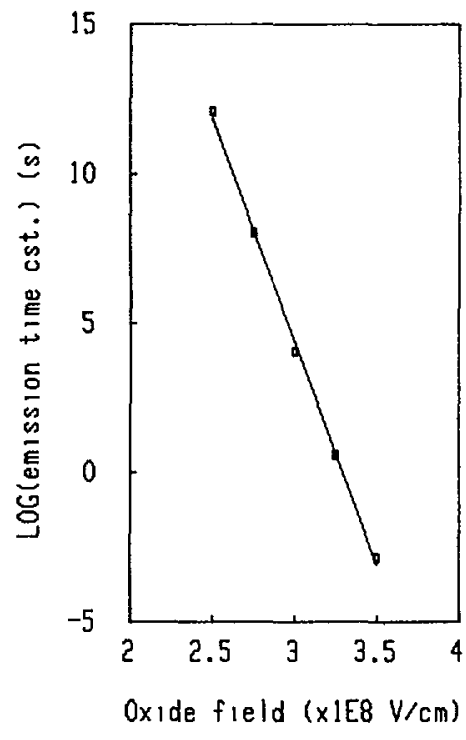

a)

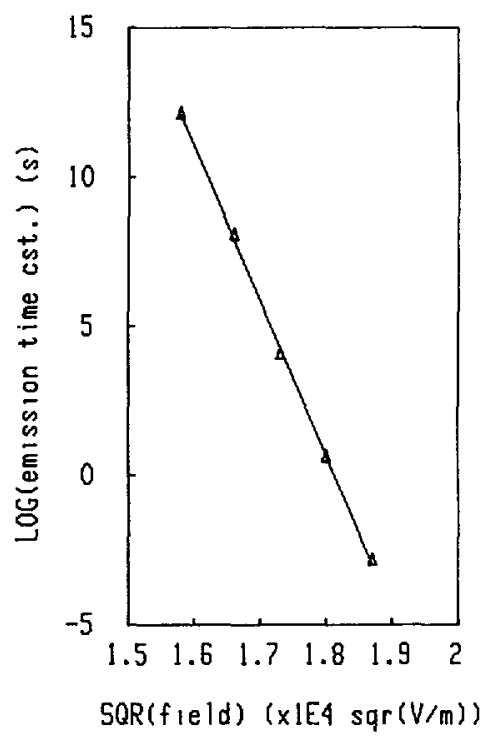

b)

Fig. 9. - Constante de temps d'émission des défauts d'oxyde induits par le vieillissement en fonction (a) du champ électrique dans l'oxyde, (b) de la racine carrée du champ (d'après Réfs. [45, 46]).

[(a) Linear of (b) square root dependence of the emission time constant of the aging-induced oxide defects versus the oxide electric field (from Refs. [46, 47]).]

c'est-à-dire une distorsion importante du réseau, et inversement. La figure 10 présente ainsi les deux configurations possibles selon la nature du défaut. Nous avons montré par les mesures de GF et de pompage de charge la nature accepteur des défauts créés par injections de porteurs chauds dans la zone de recouvrement grille-drain [43, 44] (voir Sect. 3 et 4). Nous pensons donc que la configuration défaut accepteur avec une relaxation de réseau importante est le bon modèle pour ces défauts induits par l'injection de porteurs chauds. De plus, cette configuration est en accord avec les valeurs importantes obtenues pour la section de capture des électrons par ces défauts, $\sigma_{\mathrm{e}} \approx 10^{-14} \mathrm{~cm}^{2}$, qu'ils aient été créés à $V_{\mathrm{g}}=V_{\mathrm{d}} / 4$ ou $V_{\mathrm{g}}=V_{\mathrm{d}}[47,48]$ (voir Sect. 3.2). En effet, dans le modèle de capture par émission multiphonon, et dans le cas où : i) $d_{\mathrm{FC}}$ est large i.e. $d_{\mathrm{FC}} \approx E_{\mathrm{T}}$, ii) à haute température tel que $k T>\hbar \omega / 2[72]$, on peut déterminer l'énergie d'activation de la section de capture $E_{\mathrm{b}}$ par : $E_{\mathrm{b}}=\left(E_{\mathrm{T}}-d_{\mathrm{FC}}\right)^{2} / 4 d_{\mathrm{FC}}$. Avec les valeurs déterminées ci-dessus (Fig. 10) pour un défaut accepteur, cela conduit à $E_{\mathrm{b}} \approx 30 \mathrm{meV}$ donnant alors une section de capture $\sigma_{e}=\sigma_{\infty} \exp \left(-E_{b} / k T\right)$ importante, la barrière d'énergie pour la capture étant quasiment nulle [62].

L'identification de ce défaut est assez difficile à ce stade de l'étude. On peut tenter de discuter les propriétés électriques et optiques que nous avons obtenues par rapport aux principaux défauts connus dans $\mathrm{SiO}_{2}$. liaison pendante de $\mathrm{Si}\left(\mathrm{O}_{3} \equiv \mathrm{Si}\right)$, centre $\mathrm{E}^{\prime}$ (liaison pendante de Si chargée positivement sur une lacune d'oxygène), liaison pendante d'oxygène $\left(\mathrm{O}_{3} \equiv \mathrm{Si}-\mathrm{O}^{\circ}\right)$ et le radical peroxyl $\left(\mathrm{O}_{3} \equiv \mathrm{Si}-\mathrm{O}-\mathrm{O}^{\circ}\right)$, tous identifiés par RPE [73-77]. Les niveaux d'énergie de ces défauts ont été calculés par Robertson et al. [78, 79], et seul $\left(\mathrm{O}_{3} \equiv \mathrm{Si}^{*}\right)$, pourrait correspondre à notre niveau accepteur à $\sim 1,7 \mathrm{eV}$. Les calculs de densités locales donnent en effet des niveaux à $\sim 7 \mathrm{eV}$ et $\sim 4,9 \mathrm{eV}$ par rapport à la bande de valence 


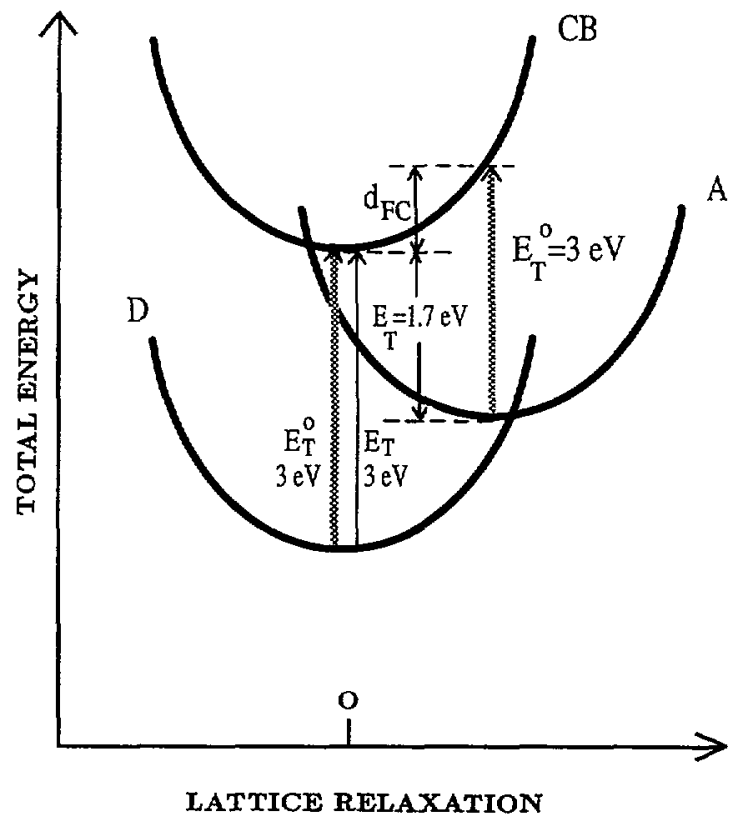

Fig. 10. - Diagramme de configuration des défauts d'oxyde induits par le vieillissement (d'après Réfs. $[46,47])$.

[Coordinate configuration diagram of the oxide defects induced by the aging (from Refs. [46, 47]).]

pour les états de charge - et 0 . Cela correspond à une énergie d'ionisation thermique de $\sim 3 \mathrm{eV}$ pour la transition $0 /-$, à comparer à notre détermination experimentale de $\sim 1,7 \mathrm{eV}$. Par ailleurs, la transition optique $\mathrm{a} \sim 2 \mathrm{eV}$, observée dans les spectres d'absorption, a été attribuée à l'état de charge - de ce défaut [73, 79]. Cette valeur est relativement proche du seuil que nous observons par spectroscopie de photodépopulation. Les autres défauts présentent des niveaux (calculés) et des transitions optiques plus en profondeur vers la moitié inférieure de la bande interdite de $\mathrm{SiO}_{2}$. Une identification réelle des défauts induits par les porteurs chauds au-dessus du drain demande une étude de type RPE qui ne pourra éventuellement être possible que par la méthode de SDR (ou EDMR, Electrically Detected Magnetic Resonance) appliquée sur des MOSFET (voir Sect. 7) [40-42, 80-83].

\section{Modèle de dégradation des n-MOSFET LDD.}

Après avoir présenté les propriétés physiques des défauts induits par l'injection de porteurs chauds, propriétés obtenues par des méthodes de caractérisation des défauts dans les MOSFET submicroniques (et plus particulièrement, par une nouvelle approche utilisant la technique de GF, et des mesures optiques), nous allons examiner les conditions de création des défauts en fonction des conditions de vieillissement [49]. La création des défauts sera corrélée avec les dégradations des $I-V$ mesurées expérimentalement, ainsi que par une simulation $2 \mathrm{D}$ dans laquelle nous prendrons en compte ces différents défauts. Les paramètres des défauts extraits des mesures de pompage de charge et de GF seront pris comme données d'entrée pour le simulateur IMPACT $[29,51-54]$ et les caractéristiques $I-V$ ainsi simulés seront comparés aux caractéristiques $I-V$ expérimentales afin d'avoir une approche autocohérente des interactions défauts-caractéristiques électriques des composants. 
Les paramètres technologiques des transistors LDD sont les suivants :

$* L=1 \mu \mathrm{m}, W=100 \mu \mathrm{m} \quad\left(L_{\text {eff }}=0,85 \mu \mathrm{m}\right), t_{\mathrm{ox}}=200 \AA \AA$, oxyde sec, grille poly-Si $\mathbf{n}^{+}$

* recouvrement grille-drain $0,06 \mu \mathrm{m}$, espaceur $0,25 \mu \mathrm{m}$,

* implantation $S / D$;

$\mathrm{n}^{-} \quad 2 \times 10^{13} \mathrm{~cm}^{-2} / 15 \mathrm{keV} ; \mathrm{n}^{+} \cdot 5 \times 10^{15} \mathrm{~cm}^{-2} / 40 \mathrm{keV}$

* ajustement de seuil : $B=1,6 \times 10^{12} \mathrm{~cm}^{-2} / 25 \mathrm{keV}$,

* substrat : $5 \times 10^{16} \mathrm{~cm}^{-3}$, type $\mathrm{p}$.

Les vieillissements sont effectués à $V_{\mathrm{d}}=8,5 \mathrm{~V}$ et pour 4 conditions de tensions de grille : $V_{\mathrm{g}}=V_{\mathrm{d}}, V_{\mathrm{d}} / 2, V_{\mathrm{d}} / 4$ et $V_{\mathrm{d}} / 8$ durant $4 \times 10^{4} \mathrm{~s}$. Après vieillissement, on utilise des courtes injections d'électrons (CIE), $100 \mathrm{~s}$ à $V_{\mathrm{g}}=V_{\mathrm{d}}=8,5 \mathrm{~V}$, et des courtes injections de trous (CIT), $100 \mathrm{~s}$ à $V_{\mathrm{g}}=V_{\mathrm{d}} / 4$, pour modifier l'état de charge des défauts dans l'oxyde.

6.1 MESURE DES COURANTS DE DRAIN. - Nous mesurons la dégradation des caractéristiques $I_{\mathrm{d}}-V_{\mathrm{g}}$ en régime linéaire qui peuvent se classer en deux types. Le type-I (Fig. 11a) est observé pour les vieillissements à $V_{\mathrm{g}}=V_{\mathrm{d}}$ et $V_{\mathrm{d}} / 2$. La distorsion observée est une modification de la pente au-dessus de seuil (diminution de $g_{\mathrm{m}}$ ) et une très faible dérive de $V_{\mathrm{t}}, \Delta V_{\mathrm{t}}=12 \mathrm{mV}$ (mesuré à $I_{\mathrm{d}}=1 \mu \mathrm{A} / \mu \mathrm{m}$ ). Il $\mathrm{n}$ 'y a aucun effet d'une CIE (courbe « SEI », Fig. 11a). Une CIT ramène partiellement la courbe vers la caractéristique initiale (courbe «SHI », Fig. 11a).

Le comportement de type-II (Fig. 11b) est observé pour les vieillissements à bas $V_{\mathrm{g}}\left(V_{\mathrm{g}}=V_{\mathrm{d}} / 4\right.$ et $\left.V_{\mathrm{d}} / 8\right)$. La déformation est maintenant un changement de la pente avec une dérive de l'ensemble de la caractéristique vers les tensions positives. Une CIE, induit une dérive supplémentaire de $V_{\mathrm{t}}$ et une CIT ramène la caractéristique sur celle mesurée immédiatement après le vieillissement. La figure 12, résume l'amplitude de ces dérivées en fonction de la tension de grille de vieillissement. On observe clairement l'effet d'une CIE après les vieillissements à bas $V_{\mathrm{g}}$. Cette augmentation de la dégradation peut s'expliquer par le remplissage par des électrons de pièges accepteurs crés à l'état neutre lors du vieillissement par injection de trous. Ce résultat est similaire à ceux de Doyle et al. obtenus sur d'autres dispositifs de technologie différente [33]. Nous pensons que le modèle proposé par Heremans et al. [11] (défauts donneurs dans l'oxyde et états d'interface accepteurs) n'est pas suffisant dans le cas présent. En effet, selon ce modèle, la dérive des caractéristiques $I_{\mathrm{d}}-V_{\mathrm{g}}$ après le vieillissement et une CIE est alors uniquement due aux états d'interface, le retour vers la caractéristique initiale après une CIT provenant alors de la compensation (totale ou partielle) des états d'interface accepteurs par les donneurs dans l'oxyde chargés positivement. Les mesures de pompage de charge (voir Sect. 6.2) associées à des simulations 2D (Sect. 6.4) nous montreront que ce modèle n'explique pas totalement l'amplitude des dégradations observées et que la présence de défauts accepteurs dans la zone de recouvrement grille-drain est nécessaire dans notre cas. Les raisons possibles expliquant cette différence de comportement seront discutées ultérieurement (Sect. 6.5).

6.2 MESuRE DE POMPAGE DE CHARGE. - Un exemple typique de caractéristique $I_{\mathrm{cp}}-V_{\mathrm{b}}$ est donné à la figure 13. Les principaux résultats que l'on en extrait sont : une augmentation des états d'interface, la présence de charges positives au-dessus du canal, indiqué par le décalage $\Delta V_{\mathrm{cp}}^{+}$de l'ensemble des flancs de la caractéristique après une CIT [11, 59], et la présence de charges négatives au-dessus du drain indiqué par le décalage $\Delta V_{\mathrm{cp}}^{-}$du flanc à bas $V_{\mathrm{b}}$ après une CIE [44, 47] (voir Sect. 4). Ces résultats sont communs aux 


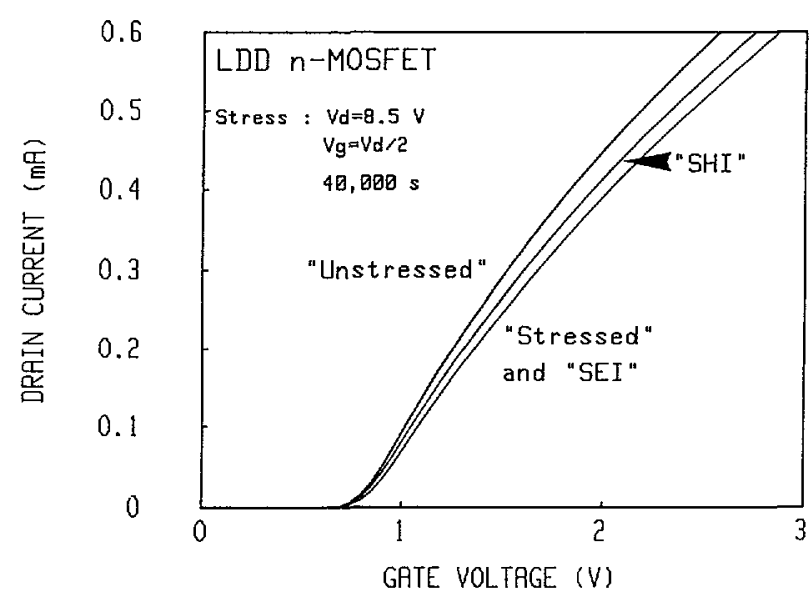

a)

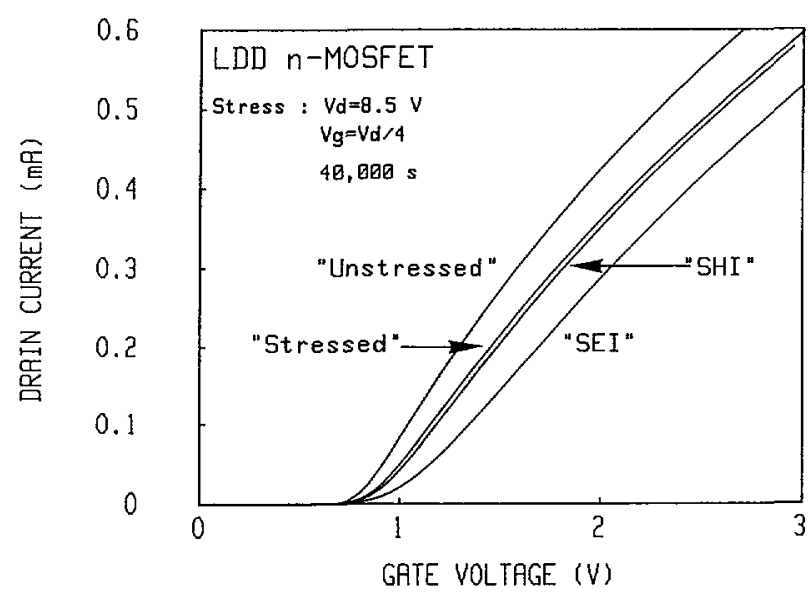

b)

Fig. 11. - Evolution des courbes $I_{\mathrm{d}}-V_{\mathrm{g}}$ après un vieillissement et des courtes injections d'électrons et de trous. (a) vieillissement à haut et moyen $V_{\mathrm{g}}$, (b) à bas $V_{\mathrm{g}}$ (d'après Réf. [49]).

[Evolutions of the $I_{\mathrm{d}}-V_{\mathrm{g}}$ curves after aging and short electron and hole injection phases. (a) aging at high and medium $V_{\mathrm{g}}$ and (b) at low $V_{\mathrm{g}}$ (from Ref. [49]).]

quatre conditions de vieillissement avec des amplitudes différentes résumées dans le tableau I. En ce qui concerne les charges positives, il faut noter que le décalage $\Delta V_{\mathrm{cp}}^{+}=-1,6 \mathrm{~V}$ correspond à des défauts pré-existants. En effet, le même résultat est observé après une CIT identique sur des MOSFET non vieillis. Il est peu probable qu'une injection de $100 \mathrm{~s}$ puisse créer une concentration $\sim 1,8 \times 10^{12}$ charges $/ \mathrm{cm}^{2}$, cela correspondrait à un taux de création largement supérieur à 1 (au lieu de $10^{-3}-10^{-2}$ mesurée par différents groupes pour la création de défauts par les trous chauds) $[11,61,84]$. Ce décalage est donc induit par le piégeage de trous sur des défauts donneurs pré-existants dans l'oxyde. Une estimation grossière de la section de capture donne $\sim 5 \times 10^{-12} \mathrm{~cm}^{2}$ [49]. Des pièges à trous avec de larges sections de capture ont souvent été observés dans les études antérieures, ils semblent 


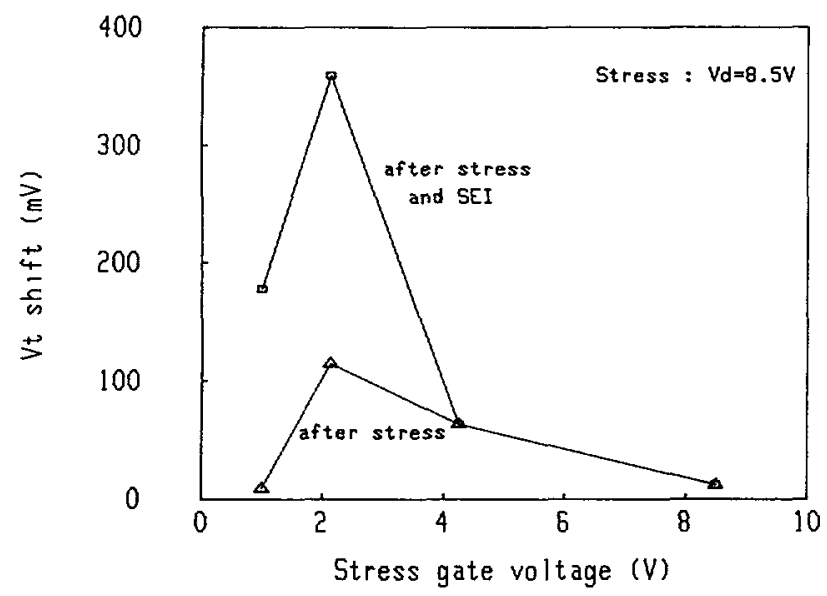

Fig. 12. - Décalage de la tension de seuil (après le vieillissement et après une CIE) en fonction de la tension de grille pendant le vieillissement (d'après Réf. [49]).

[Threshold voltage shift (after the aging and SEI) versus the gate voltage during the aging (from Ref. [49]).]

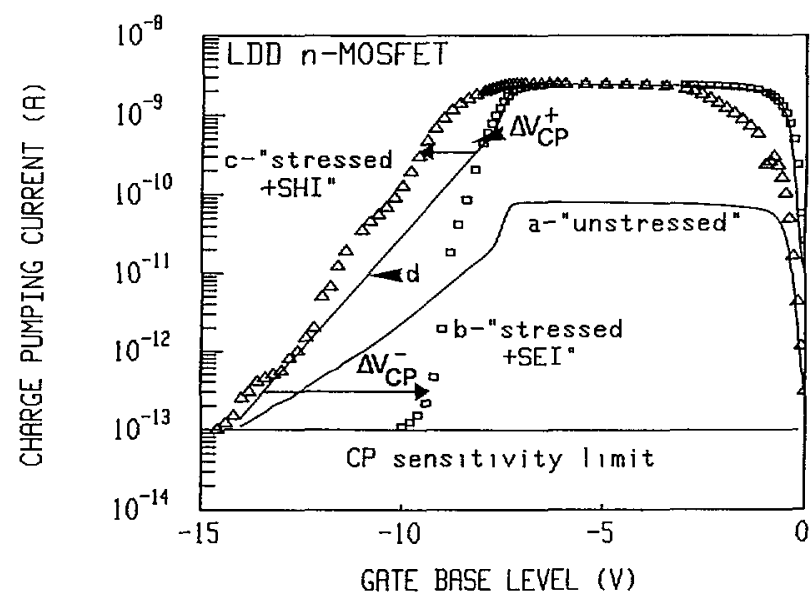

Fig. 13. - Courbes de pompage de charge : (a) MOSFET non-vieilli, (b) après un vieillissement à $V_{\mathrm{g}}=V_{\mathrm{d}} / 4$ et une CIE, (c) après une CIT. La courbe (d) est calculée en ne prenant en compte que la création des états d'interface (d'après Réf. [49]).

[Charge pumping curves : (a) virgin MOSFET, (b) aged MOSFET at $V_{\mathrm{g}}=V_{\mathrm{d}} / 4$ and SEI, (c) after SHI. Curve (d) is calculated taking into account the interface state creation alone (from Ref. [49]).]

correspondre à des défauts intrinsèques de $\mathrm{SiO}_{2}$ dont la concentration est très dépendante des conditions de fabrication [61]. Il n'y a donc pas de création de défauts donneurs $\left(\Delta N_{\text {or }}^{+1}\right)$ pour les vieillissements à haut $V_{\mathrm{g}}$, ce qui est en accord avec d'autres résultats [11]. Une CIE sur un MOSFET non vieilli n'induit aucune dérive $\Delta V_{\text {cp }}^{-}$, ce qui signifie que celles mesurées après un vieillissement résultent des défauts accepteurs $\left(\Delta N_{\mathrm{ot}}^{\mathrm{O}-}\right.$ ) créés lors de celuici. 
Tableau I. - Paramètres des défauts induits lors du vieillissement à différentes tensions de grille. Les grandeurs $\Delta I_{\mathrm{cp}}, \Delta V_{\mathrm{cp}}^{+}$et $\Delta V_{\mathrm{cp}}^{-}$sont mesurées par pompage de charge. Elles donnent les densités d'états d'interface $\Delta D_{\mathrm{it}}$, les concentrations de défauts donneurs $\Delta N_{\mathrm{ot}}^{+/ 0}$ et accepteurs $\Delta N_{\text {ot }}^{01}$ - dans l'oxyde (d'après Ref. [49]).

[Parameters of induced-defects by aging at several gate voltages. The values of $\Delta l_{\mathrm{cp}}$, $\Delta V_{\mathrm{cp}}^{+}$and $\Delta V_{\mathrm{cp}}^{-}$are obtained from charge pumping measurements. They give the interface state density $\Delta D_{\mathrm{it}}$, the concentration of donor-like $\Delta N_{\text {ot }}^{+10}$ and acceptor-like $\Delta N_{\text {of }}^{0 /}$ - oxide traps (from Ref. [49]).]

\begin{tabular}{|l|c|c|c|c|c|}
\hline & $V_{\mathrm{g}}=V_{\mathrm{d}} / 8$ & $V_{\mathrm{g}}=V_{\mathrm{d}} / 4$ & $V_{\mathrm{g}}=V_{\mathrm{d}} / 2$ & $V_{\mathrm{g}}=V_{\mathrm{d}}$ & \\
\hline$\Delta I_{\mathrm{cp}}$ & 1,75 & 2,4 & 2,6 & 0,6 & $\mathrm{nA}$ \\
$\Delta D_{\mathrm{tt}}$ & $2,4 \times 10^{11}$ & $3,3 \times 10^{11}$ & $3,6 \times 10^{11}$ & $0,3 \times 10^{10}$ & $\mathrm{~cm}^{-2} \mathrm{eV}^{-1}$ \\
$\Delta V_{\mathrm{cp}}^{+}$ & $-2,3$ & $-1,9$ & $-1,6$ & $-1,6$ & $\mathrm{~V}$ \\
$\Delta N_{\mathrm{ot}}^{+10}$ & $5 \times 10^{11}$ & $1 \times 10^{11}$ & 0 & 0 & $\mathrm{~cm}^{-2}$ \\
$\Delta V_{\mathrm{cp}}^{-}$ & 2 & 3,3 & 2,2 & 1 & $\mathrm{~V}$ \\
$\Delta N_{\mathrm{ot}}^{0 \prime}$ & $2,6 \times 10^{12}$ & $4 \times 10^{12}$ & $2,6 \times 10^{12}$ & $1,2 \times 10^{12}$ & $\mathrm{~cm}^{-2}$ \\
\hline
\end{tabular}

6.3 Mesures DE GRILle-FlotTANTE, LOCALiSATION DES DEFAuTs. - Par rapport aux résultats de base décrits section 3, deux types de comportement sont observés en fonction de la tension de grille de vieillissement. Le type I est uniquement observé pour le vieillissement à $V_{\mathrm{g}}=V_{\mathrm{d}}$ (Fig. 14a) et le type II pour les 3 autres cas de vieillissement (Fig. 14b). Dans tous les cas, nous observons une diminution de $I_{\mathrm{g}}$ et une augmentation de $I_{\text {sub }} / I_{\mathrm{d}}$ pendant le vieillissement. Toutefois, les ordres de grandeurs sont différents (Fig. 15) et la plus grande dégradation de $I_{\mathrm{g}}$ est observée pour un vieillissement à $V_{\mathrm{g}}=V_{\mathrm{d}}$. Les types I et II se distinguent par le comportement des courbes $I_{g}-V_{g}$ lors des CIE et CIT suivant le vieillissement. Dans le cas de type $I$, la courbe après une CEI est confondue avec celle mesurée immédiatement après le vieillissement. Ceci est conforme à la création de défauts accepteurs chargés négativement par la capture d'électrons (voir Sect. 3) [44]. Dans tous les autres cas (Fig. 14b) ceci n'est plus valable. Ce comportement (type II) s'explique par l'injection simultanée d'électrons et de trous, par la localisation respective des défauts donneurs et accepteurs dans l'oxyde, et par la position du point d'injection des électrons pendant la mesure du courant de grille.

L'injection simultanée d'électrons et de trous [8] implique que les donneurs pré-existants sont chargés positivement et les accepteurs créés sont chargés négativement. A partir des résultats de pompage de charge et de simulations $2 \mathrm{D}$ sur la localisation des points d'injection des électrons et des trous le long de l'interface $[25,26]$, le schéma grossier de la localisation des défauts donneurs et accepteurs, donné à la figure 16, est obtenu. Il est probable que les deux distributions se recouvrent au niveau de la jonction drain-canal. Pour analyser le comportement des caractéristiques $I_{\mathrm{g}}-V_{\mathrm{g}}$ nous devons connaître quelle est la zone analysée lors de la mesure de GF. Des simulations 2D [5, 27, 85] et des simulations Monte Carlo [32] ont montré que le point d'injection des électrons se déplace vers le canal quand $V_{\mathrm{g}}$ diminue. L'amplitude de ce déplacement est plus ou moins important, des valeurs entre 


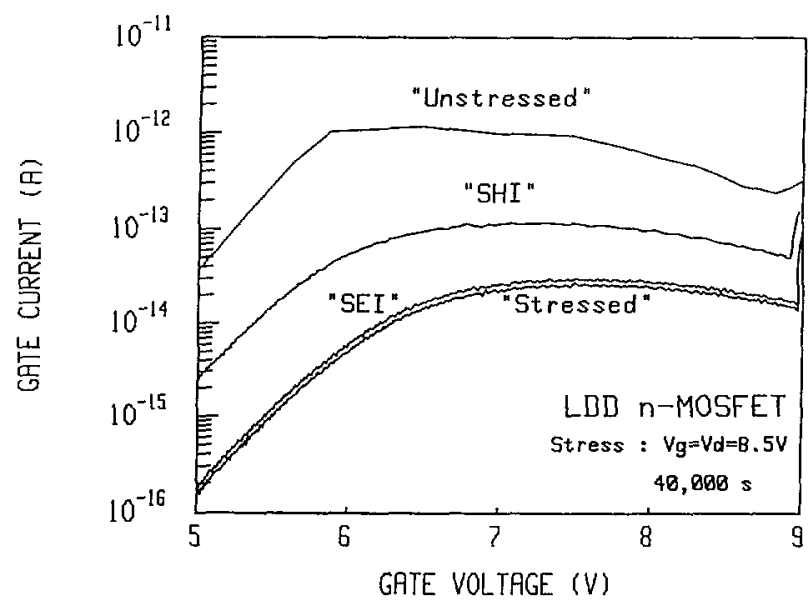

a)

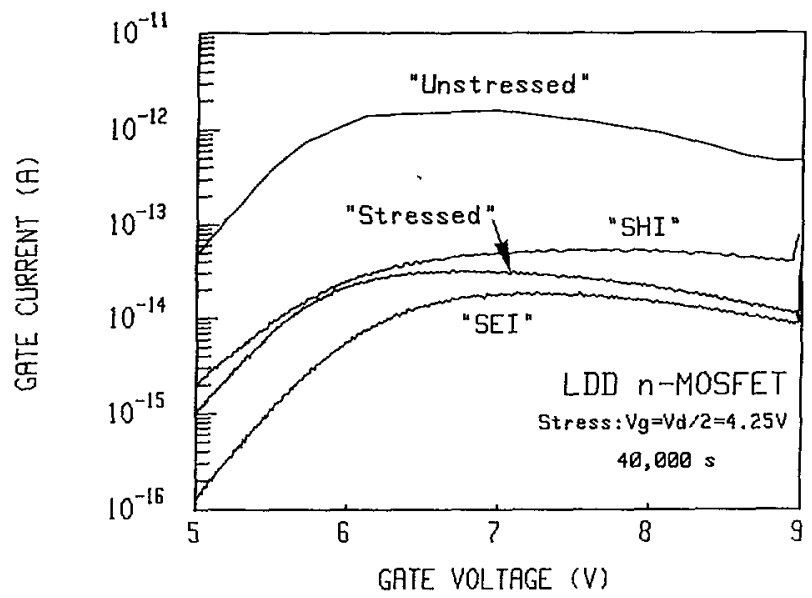

b)

Fig. 14. - Caractéristiques du courant de grille d'un MOSFET vieilli et effets des CIE et CIT : (a) vieillissement à haut $V_{g}$, (b) à moyen et bas $V_{\mathrm{g}}$ (d'après Réf. [49]).

[Gate current characteristics of an aged MOSFET and effects of SEI and SHI phases : (a) aging at high $V_{\mathrm{g}}$, (b) at medium and low $V_{\mathrm{g}}$ (from Ref. [49]).]

0,02 et $0,15 \mu \mathrm{m}$ ont été données. De plus, il a été montré qu'en régime linéaire $\left(V_{\mathrm{g}}>V_{\mathrm{d}}\right)$ le point d'injection est situé plus en profondeur au-dessus du drain [85]. Il est donc raisonnable de penser que la branche linéaire de la caractéristique $I_{\mathrm{g}}-V_{\mathrm{g}}$ mesurée par GF est essentiellement sensible aux défauts accepteurs, et la branche saturée à la zone où les deux types de défauts coexistent (Fig. 16). Ceci permet d'expliquer simplement le comportement de type II (Fig. 14b). Après le vieillissement, des charges positives et négatives sont induites comme montré sur la figure 16. Les mesures de pompage de charge montrant que $N_{\mathrm{ot}}^{0 /-}>N_{\text {ot }}^{+10}$ (Sect. 3 et Tab. I), l'ensemble de la caractéristique $I_{\mathrm{g}}-V_{\mathrm{g}}$ décroît (courbe « stressed », Fig. 14b). Une CIE neutralise les charges positives des donneurs et conduit à une augmentation de la charge négative vue par la branche saturée de la caractéristique 


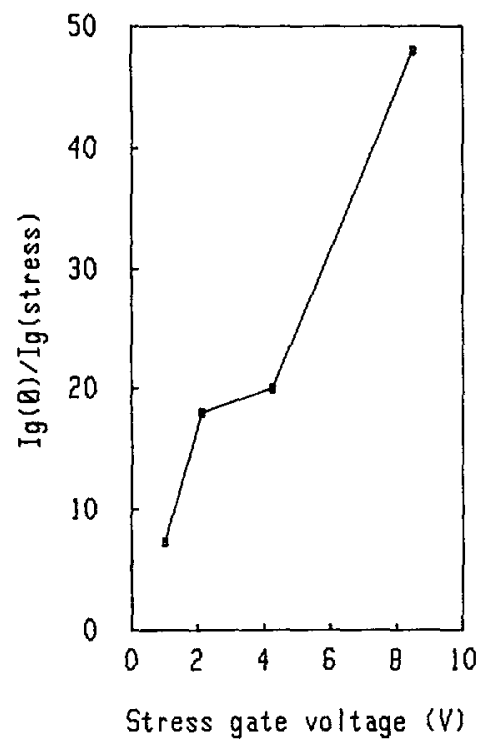

a)

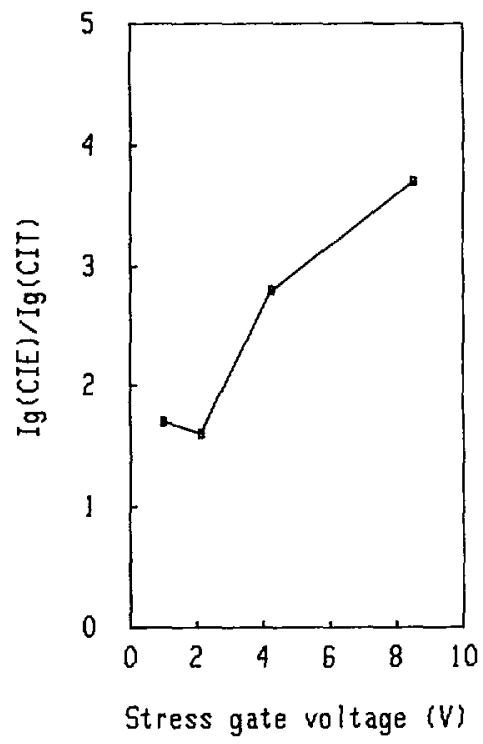

b)

Fig. 15. - Evolution avec la tension de grille de vieillissement des rapports des maximum des courants de grille : (a) avant et après vieillissement, (b) après une CIE et une CIT (d'après Réf. [49]).

[Dependence of the gate current ratio versus the gate voltage during the aging: (a) gate currents after and before the aging, (b) gate currents after SEI and after SHI (from Ref. [49]).]

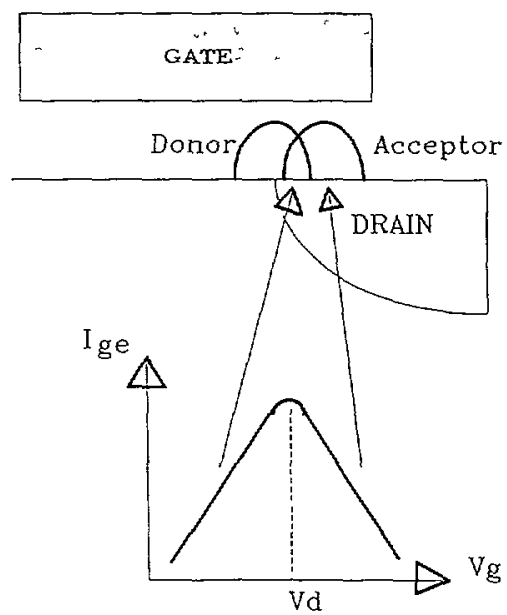

Fig. 16. - Représentation schématique de la localisation des défauts et influences sur les différentes zones de la caractéristique $I_{\mathrm{g}}-V_{\mathrm{g}}$ mesurée par grille-flottante (d'après Réfs. [47, 49]).

[Schematic view of the defect localization and their effects on the different parts of the $I_{\mathrm{g}}-V_{\mathrm{g}}$ characteristic measured by floating-gate technique (from Refs. [47, 49]).] 
$I_{\mathrm{g}}-V_{\mathrm{g}}$, qui voit donc son niveau de courant diminué comme le montre la courbe «SEI » de la figure $14 \mathrm{~b}$. Une CIT restaure la charge positive des donneurs et compense partiellement la charge négative des accepteurs. En conséquence, la branche saturée de la courbe $I_{\mathrm{g}}-V_{\mathrm{g}}$ retourne à sa position et la branche linéaire augmente à cause de la réduction des charges négatives au-dessus du drain (courbe «SHI », Fig. 14b). Bien que grossière, cette description phénoménologique permet de comprendre l'ensemble des phénomènes observés par les mesures de GF.

Finalement, la figure 15 suggère que la plus forte création de défauts accepteurs a lieu pour le vieillissement à $V_{\mathrm{g}}=V_{\mathrm{d}}$. Ceci semble en contradiction avec les mesures de pompage de charge (Tab. I) qui montrent un maximum de création pour $V_{\mathrm{g}}=V_{\mathrm{d}} / 4$. En réalité, ceci indique que la localisation des défauts accepteurs créés à $V_{\mathrm{g}}=V_{\mathrm{d}}$ est plus en profondeur audessus du drain, et que seule une fraction est détectable par les mesures de pompage de charge. Ceci est en accord avec le déplacement du point d'injection des électrons en fonction de $V_{\mathrm{g}}$ obtenu par les simulations 2D et Monte Carlo [15, 27, 32, 85].

6.4 Simulations 2D. - La cohérence entre les résultats obtenus par les techniques de GF et pompage de charge sur les défauts induits par les porteurs chauds et les dérives des caractéristiques $I_{\mathrm{d}}-V_{\mathrm{g}}$ est vérifiée par des simulations 2D [49]. Nous avons utilisé le simulateur IMPACT (Isen Modelling PAckage for Cmos Technology) [51, 52] qui permet de prendre en compte des distributions localisées de défauts dans l'oxyde et à l'interface [53, 54]. Dans le cas présent, les états d'interface et les donneurs dans l'oxyde sont modélisés par une distribution gaussienne dans le canal à $0,07 \mu \mathrm{m}$ du drain et les défauts d'oxyde accepteurs sont modélisés par une gaussienne au-dessus du drain, centrée sur la position du maximum de champ latéral. Les densités de défauts inclues dans le simulateur sont celles extraites des mesures de pompage de charge (Tab. I). Pour montrer le rôle nécessaire des défauts d'oxyde accepteurs, on compare les $I_{\mathrm{d}}-V_{\mathrm{g}}$ simulés dans deux conditions : i) création d'états d'interface uniquement, ii) création d'états d'interface et de défauts accepteurs dans l'oxyde au-dessus du drain, avec les $I_{\mathrm{d}}-V_{\mathrm{g}}$ mesurés après vieillissement et application d'une CIE. Dans ce cas, les donneurs pré-existants sont neutres et ne sont donc pas pris en considération. Deux cas de vieillissement sont montrés figures 17 et 18 , respectivement pour $V_{\mathrm{g}}=V_{\mathrm{d}}$ et $V_{\mathrm{d}} / 4$. Ces résultats démontrent clairement que des défauts d'oxyde de type accepteur sont nécessaires pour expliquer la dégradation de nos transistors. Une conclusion similaire a été donnée récemment par d'autres auteurs pour des MOSFET conventionnels [28, 33, 86]. La figure 19 montre que la cohérence de notre modèle de dégradation est correcte pour l'ensemble des conditions de vieillissement et que ces défauts de type accepteur sont les principaux responsables de la dégradation des MOSFET utilisés dans cette étude [49]. Il est toutefois surprenant qu'une faible variation de $N_{\text {ot }}$ entraîne des variations très importantes de $\Delta V_{\mathrm{t}}$. Ceci est expliqué par la figure 20 qui montre l'évolution simulée de $V_{\mathrm{t}}$ en fonction de la concentration des défauts accepteurs. La relation est fortement non linéaire et elle permet d'induire la notion de seuil de tolérance pour la création de ces défauts. Ce concept peut être utilisé pour analyser, uniquement au moyen de simulations $2 \mathrm{D}$, la fiabilité (sensibilité aux dégradations) de différentes technologies. De plus, il pourrait être utilisé pour définir une durée de vie des MOSFET, pourvu que les cinétiques de création des défauts soit correctement connues.

6.5 Discussions. - Il ressort de cette étude que les défauts de type accepteur localisés audessus du drain dans l'oxyde de recouvrement grille-drain sont particulièrement importants pour expliquer les mécanismes de dégradation de nos dispositifs MOSFET LDD. Ceci est en accord avec les travaux de Doyle et al. obtenus pour des structures non-LDD et MDD [33] mais en désaccord avec ceux de Heremans et al. également sur des structures non-LDD [11]. 


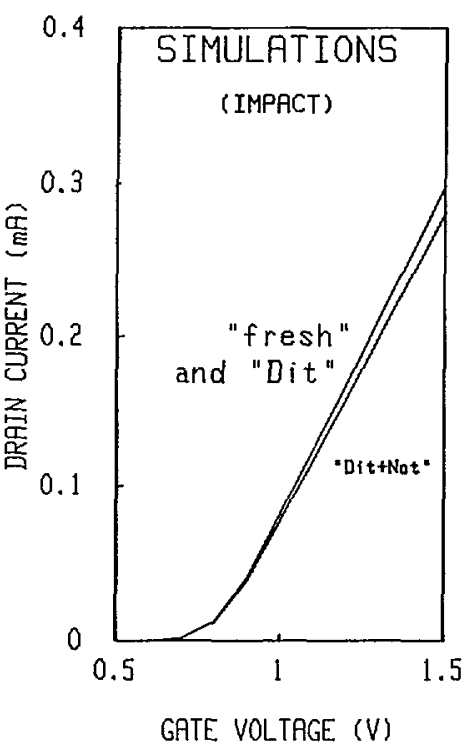

a)

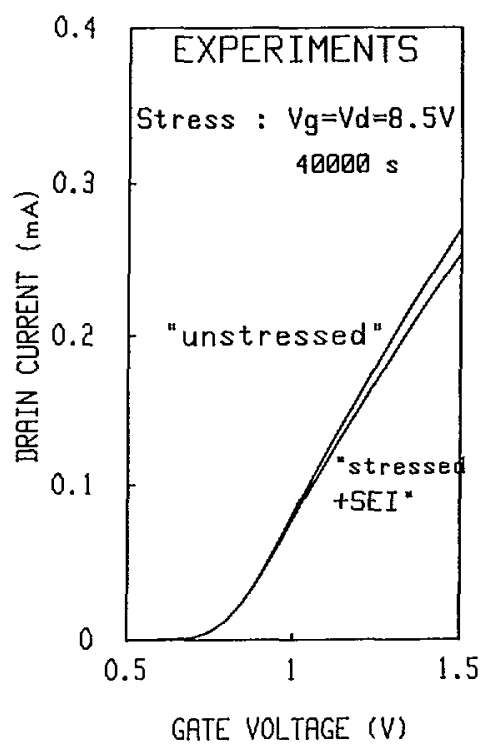

b)

Fig. 17. - Comparaison des mesures et des simulations 2D des courbes $I_{\mathrm{d}}-V_{\mathrm{g}}$ prenant en compte l'influence des états d'interface et/ou des charges négatives d'oxyde au-dessus du drain, pour un vieillissement à haut $V_{g}$ (d'après Réf. [49]).

[Comparison of the measured and the 2D simulated $I_{d}-V_{g}$ curves taking into account the interface states and/or the negative oxide charges located above the drain for aging at high $V_{\mathrm{g}}$ (from Ref. [49]).]

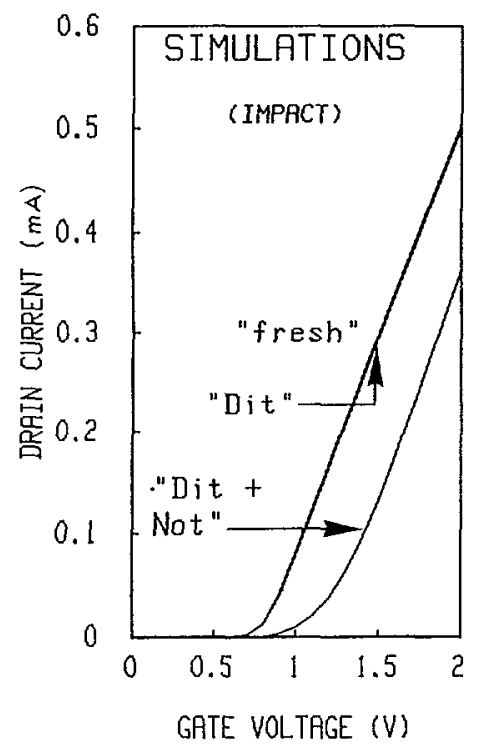

a)

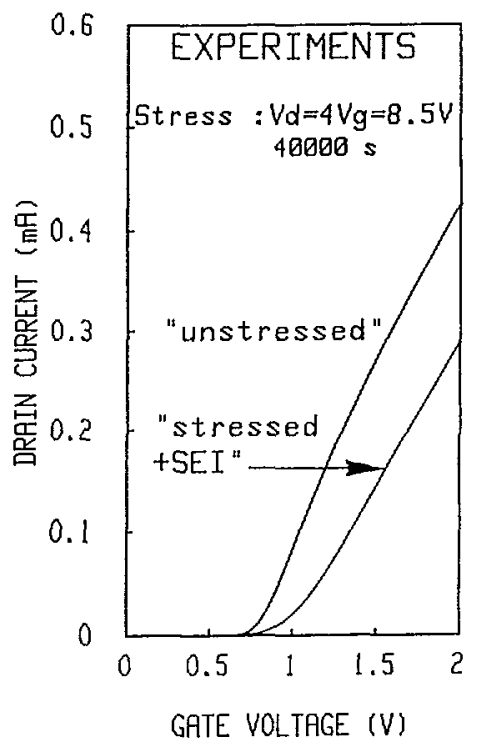

b)

Fig. 18. - Même résultat que la figure 17 pour un vieillissement à bas $V_{\mathrm{g}}$ (d'après Réf. [49]).

[Same as in figure 17 for aging at low $V_{\mathrm{g}}$ (from Ref. [49]).] 


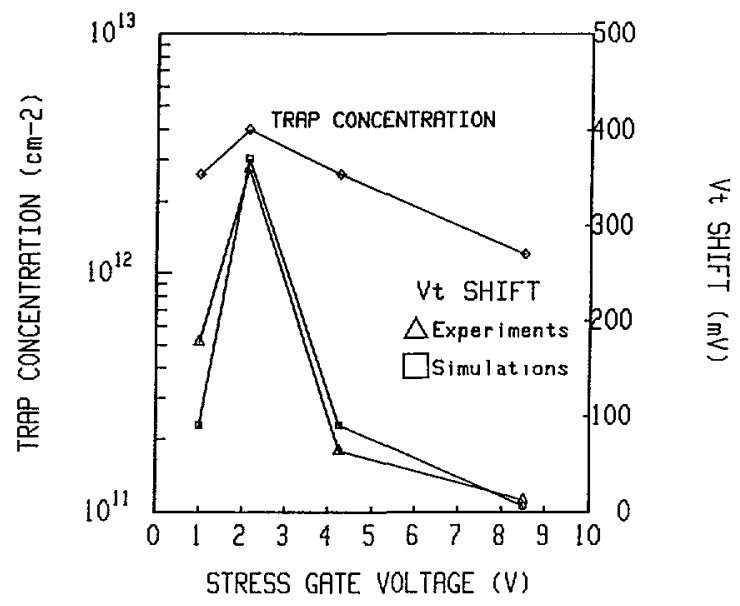

Fig. 19. - Comparaison des dérives de tensions de seuil mesurées et simulées en tenant compte des concentrations de charges négatives extraites des mesures de pompage de charge pour différentes conditions de vieillissement (d'après Réf. [49]).

[Comparaison of the measured and 2D simulated threshold voltage shifts taking into account the oxide negative charges measured by charge pumping for different aging conditions (from Ref. [49]).]

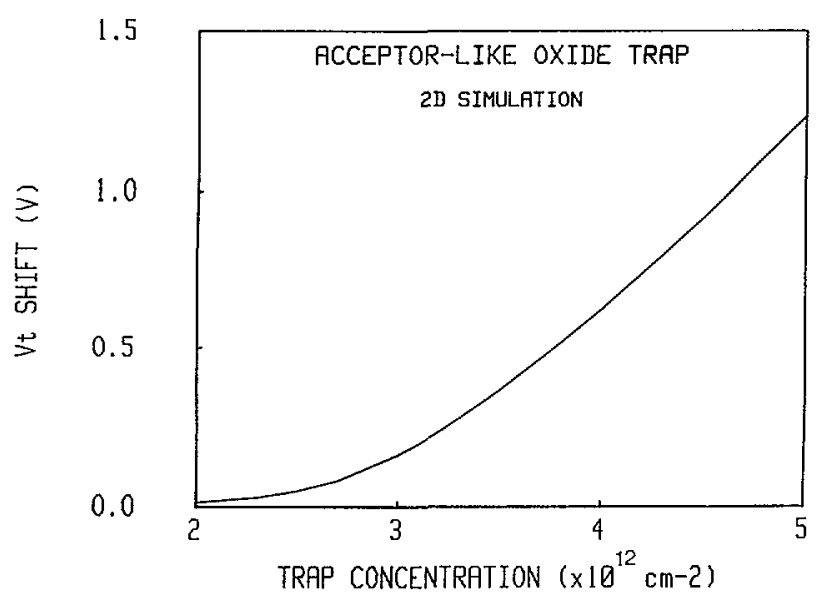

Fig. 20. - Simulation 2D de l'évolution de la tension de seuil en fonction de la concentration de charges négatives dans l'oxyde au-dessus du drain (d'après Réf. [49]).

[2D simulation of the threshold voltage shift versus the amount of negative charges located in the oxide above the drain (from Ref. [49]).]

Diverses raisons peuvent être évoquées pour expliquer ce désaccord entre les différents résultats.

* Dans le cas de structures LDD, il est connu que le phénomène de piégeage d'électrons est relativement plus important que dans les structures conventionnelles [30-32].

* Dans notre cas, la tension de drain lors des vieillissements est assez élevée $(8,5 \mathrm{~V})$ ce qui correspond à un champ perpendiculaire dans l'oxyde au-dessus du drain de $\sim 3,2 \mathrm{MV} / \mathrm{cm}$ pour 
les vieillissements à $V_{\mathrm{g}}=V_{\mathrm{d}} / 4$. Ce champ est respectivement de $\sim 1,3 \mathrm{MV} / \mathrm{cm}$ et $\sim 1,7 \mathrm{MV} / \mathrm{cm}$ dans les expériences de Doyle $e t$ al. et Heremans et al. [11, 33]. Notre champ est proche du seuil de création mesuré par des expériences d'injections homogènes d'électrons $(\sim 4 \mathrm{MV} / \mathrm{cm})$ [87]. De plus, nos durées de vieillissement sont plus longues que celles utilisées par Heremans et al., ce qui implique une création plus importante de défauts accepteurs qui possèdent des taux de création de l'ordre de $10^{-9}$ pour la création par injection d'électrons et de $10^{-3}-10^{-2}$ pour une création par injection de trous [49]. Toutefois, ces arguments ne s'appliquent pas pour expliquer la différence entre les résultats de Doyle et al. et de Heremans et al.

* Dans le cas de structures LDD, la qualité de l'oxyde près de l'espaceur est primordiale vis-à-vis des phénomènes de création des défauts accepteurs. Un exemple typique est donné à la figure 21, pour deux technologiques LDD de même géométrie, vieilli dans des conditions identiques $\left(V_{\mathrm{g}}=V_{\mathrm{d}} / 4\right)$. Une nette différence de comportement est observée par GF pour la création des défauts accepteurs, alors que le même comportement est observé par pompage de charge et sur les caractéristiques $I_{\mathrm{d}}-V_{\mathrm{g}}$ pour les deux dispositifs [47].
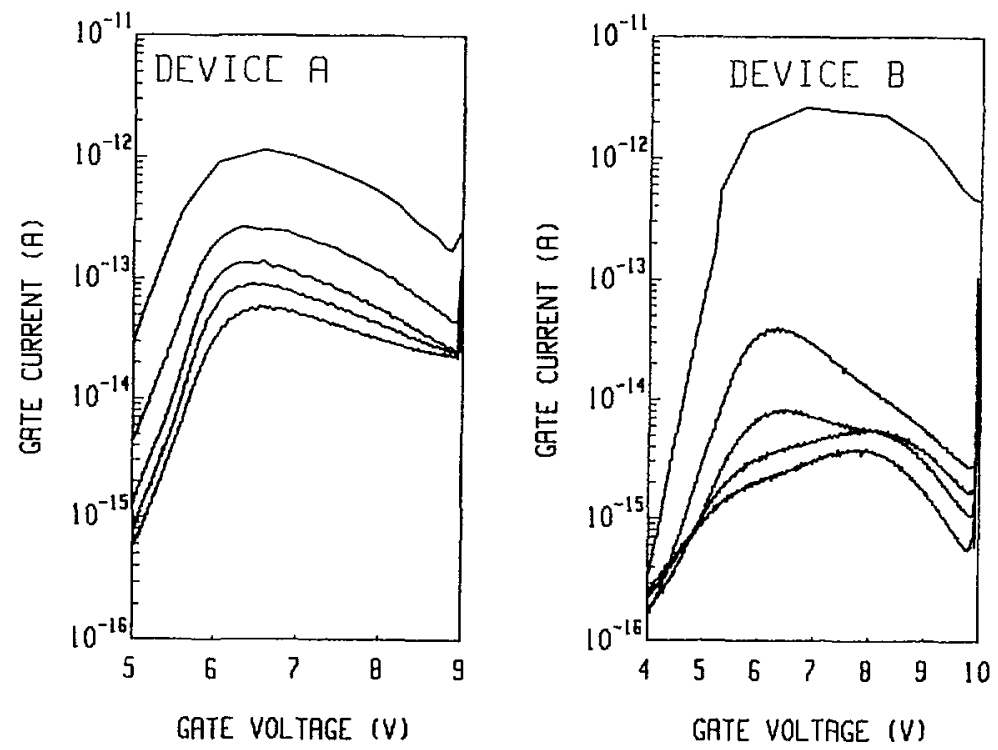

Fig. 21. - Evolution de la caractéristique $I_{\mathrm{g}}-V_{\mathrm{g}}$ pour deux MOSFET LDD de même géométrie vieillis dans les mêmes conditions, $V_{\mathrm{g}}=V_{\mathrm{d}} / 4$ avec $V_{\mathrm{d}}=8,5 \mathrm{~V}, 0,100,1000,10000$ et $40000 \mathrm{~s}$ (d'après Réf. [47]).

[Evolution of the $I_{\mathrm{g}}-V_{\mathrm{g}}$ characteristics of two MOSFET of the same dimensions aged under the same conditions, $V_{\mathrm{g}}=V_{\mathrm{d}} / 4$ with $V_{\mathrm{d}}=8.5 \mathrm{~V}, 0,100,1000,10000$ and $40000 \mathrm{~s}$ (from Ref. [47]).]

Enfin signalons des travaux très récents confirmant nos conclusions sur la création de défauts accepteurs dans l'oxyde. Des mesures de dérives des caractéristiques C-V entre grille et drain [88] et des mesures de fluctuations du courant de drain (RTS, Random Telegraph Signal) induites par des défauts isolés dans des MOSFET de très petites géométries $(0,35 \mu \mathrm{m} \times 0,5 \mu \mathrm{m})$ [89] ont clairement mis en évidence la nature accepteurs des défauts induits lors du vieillissement des MOSFET par injections de porteurs chauds. 


\section{Bilan et perspectives.}

Nos études ont essentiellement porté sur la caractérisation des propriétés physiques des défauts accepteurs induits dans l'oxyde de grille par l'injection de porteurs chauds (électrons et trous). Ces défauts avaient été moins mis en évidence dans les études antérieures, bien que leur existence ait été plusieurs fois suggérée (voir Sect. 2). Ceci est dû à leur localisation audessus du drain dans la zone de recouvrement entre grille et drain. Nos principales contributions sont les suivantes :

1) Nous avons utilisé une technique originale pour analyser ces défauts, en étudiant leurs effets sur les courants de grille très faibles du MOSFET $\left(I_{\mathrm{g}}<10^{-12} \mathrm{~A}\right)$ mesurés par une technique de grille-flottante $[44,47]$. Nous avons montré que cette technique de grilleflottante permet également de distinguer la création des états d'interface de celle de ces défauts dans l'oxyde $[47,50]$. Cette technique a également été appliquée pour la mesure des défauts dans le p-MOSFET [90,91].

2) Nous avons montré comment la technique de pompage de charge permet de caractériser ces défauts localisés au-dessus du drain, et avec quelles limites [43].

3) La mesure des propriétés optiques et électriques de ces défauts a permis d'estimer à $\sim 1,7 \mathrm{eV}$ la position du niveau d'énergie associé à la transition $0 /$ - Nous avons montré que cette transition est caractérisée par une amplitude importante de la relaxation de réseau conduisant à une transition optique de $\sim 3 \mathrm{eV}$ et à une large section de capture pour les électrons $\sim 10^{-14} \mathrm{~cm}^{2}$ [45-48]. Nous avons suggéré que ces défauts pourraient être associés à la liaison pendante de silicium $\left(\mathrm{O}_{3} \equiv \mathrm{Si}^{-}\right)$dans l'oxyde.

4) L'influence de ces défauts sur les caractéristiques électriques $I-V$ des MOSFET a été analysée, et un modèle cohérent a été établi par un couplage des caractérisations de défauts avec des simulations 2D [49]. Pour nos dispositifs LDD, nous montrons que ces défauts accepteurs sont totalement responsables des dérives des caractéristiques $I-V$ pour toutes les conditions de vieillissement [49].

Au niveau des perspectives, deux aspects nous semblent intéressants.

1) Il est primordial de préciser la compréhension fine des résultats donnés par la technique de grille-flottante. Cela inclut des aspects de localisation spatiale des défauts et des aspects plus quantitatifs (concentration). Cela demande des comparaisons des caractéristiques de courant de grille entre dégradations localisées et uniformes, et surtout un couplage étroit avec des simulations 2D des caractéristiques $I_{\mathrm{g}}-V_{\mathrm{g}}$ tenant compte des défauts. Cela impose un travail de fond sur la modélisation des injections de porteurs en présence de défauts dans le MOSFET.

2) Comme dans le cas des injections homogènes [5], une identification des défauts requiert 1'utilisation de technique SDR, également nommée EDMR [40-42, 80-83] sur les MOSFET vieillis. Une première approche en ce sens a été démontrée récemment [42] et elle nous semble très prometteuse. Nos premiers résultats au laboratoire sont encourageants.

\section{Remerciements.}

L'auteur tient à remercier A. Zylbersztejn et A. Boudou pour l'avoir accueilli dans leur groupe de recherche sur la fiabilité des composants au centre de recherche de BULL S.A. Les contributions de J. C. Marchetaux, A. Bravaix, M. Bourcerie, B. S. Doyle ont été déterminantes pour l'obtention des résultats présentés. L'auteur remercie ses collègues du département de micro-électronique de l'ISEN (et particulièrement E. Dubois et P. E. Lippens) pour les développements en simulations $2 \mathrm{D}$ de composants MOSFET. 
Ces travaux ont reçu le soutien financier du GCIS et de BULL S.A. Cet article de revue est extrait du mémoire présenté par l'auteur à l'Université des Sciences et Techniques de Lille Flandres Artois pour l'obtention de l'habilitation à diriger des recherches.

\section{Bibliographie}

[1] Kawamoto Y. et al., Symp. on VLSI Tech. (The Japan Soc. of Appl. Phys., Honolulu, 1990) p. 13 ;

YAMADA T. et al., Dig. Tech. Papers IEEE ISSCC (1991) 108 ;

MORI S. et al., ibid. 110 ;

TAGUCH et al., ibid. 112 ;

OOWAKI Y. et al., ibid. 114.

[2] Chung J. E., Jeng M., Moon J. E., Ko P. K. et Hu C., IEEE Trans. Electron. Dev. 37 (1990) 1651.

[3] Chung J. E., Jeng M., Moon J. E., Ko P. K. et Hu C., IEEE Trans. Electron. Dev. 38 (1991) 545.

[4] Tam S., Hsu F. C., Hu C., Muller R. S. et Ko P. K., IEEE Electron. Dev. Lett. EDL-4 (1983) 249.

[5] Vuillaume D., J. Phys. III France, Juin 1992.

[6] Sabnis A. G. et Nelson J. T., IEDM Tech. Digest (1985) 52.

[7] Hofmann K. R., Weber W., Werner C. et Dorda G., IEDM Tech. Digest (1984) 104.

[8] Hofmann K. R., Weber W., Werner C. et Dorda G., IEEE Trans. Electron. Dev. ED-32 (1985) 691.

[9] Lal S. K., Appl. Phys. Lett. 39 (1981) 58.

[10] Lai S. K., J. Appl. Phys. 54 (1983) 2540.

[11] Heremans P., Bellens R., Groeseneken G. et Maes H. E., IEeE Trans. Electron. Dev. ED-35 (1988) 2194.

[12] Bourcerie M., Doyle B. S., Marchetaux J. C., Boudou A. et Mingam N., ieEe Electron. Dev. Lett. EDL-10 (1989) 132.

[13] Choi J. Y., Ko P. K., Hu C. et Scott W., J. Appl. Phys. 65 (1989) 354.

[14] ORLowsKi M. K. et WeRner C., IEEE Trans. Electron. Dev. ED-36 (1989) 382.

[15] Orlowski M. K., Mazure C., Lill A., Mulhoff H. M., Hansch W., Schwerin A. et NEPPL F., Proc. ESSDERC'89, A. Heuberger, H. Ryssel et P. Lange Eds. (Springer-Verlag, Berlin, 1989) p. 711.

[16] PoOrter T. et Zoesrbergen P., IEDM Tech. Digest (1986) p. 378.

[17] SCHWERIN A., HAENSCH W. et WeRnER W., IEEE Trans. Electron. Dev. ED-34 (1987) 2493.

[18] Doyle B. S., Bourcerie M., Marchetaux J. C. et Boudou A., IEEE Trans. Electron. Dev. 37 (1990) 744.

[19] Tsuchiya T., Kobayashi T. et Nakajima S., IEEE Trans. Electron. Dev. ED-34 (1987) 386.

[20] Doyle B. S., Bourcerie M., Marchetaux J. C. et Boudou A., Proc. ESSDERC'87, P. G. Calzolari et G. Soncini Eds. (Tecnoprint, Bologne, 1987) p. 155.

[21] Annunziata R., Dalla Libera G., Ghio E. et Maggia A., Proc. ESSDERC'89, A. Heuberger, H. Ryssel et P. Lange Eds. (Springer-Verlag, Berlin, 1989) p. 715.

[22] Gabon B. et Ghibaudo G., Phys. Status Solidi A 107 (1988) 393.

[23] Reimbold G., Paviet-Salomon F., Haddara H., Guegan G. et Cristoloveanu S., Proc. ESSDERC'88, J. P. Nougier et D. Gasquet Eds. (Les Editions de Physique, France, 1988) p. 665.

[24] Acovic A., Thèse, Ecole Polytechnique de Lausanne (1989) non publié ;

Acovic A., Dutort M. et Ilegems M., IEEE Trans. Electron. Dev. 37 (1990) 1467.

[25] BABA S., KitA A. et UEDA J., IEDM Tech. Digest (1986) 734.

[26] Garrigues M., Alexandre A., Rojo P., Pedron T., Belhadda K. et Poncet A., Proc. ESSDERC'88, J. P. Nougier et D. Gasquet Eds. (Les Editions de Physique, France, 1988) p. 673. 
[27] Chen Y. et Nang T., IEEE Trans. Electron. Dev. 35 (1988) 2180.

[28] Bergonzoni C. et Doyle B. S., Proc. ESSDERC'87, P. U. Calzolari et G. Soncini Eds. (Technoprint, Bologne, 1987) p. 721.

[29] LIPPENS P. E., Travaux de simulation 2D avec le code IMPACT, communication privée.

[30] Hsu F. C. et GRINOldS H. R., IEEE Electron. Dev. Lett. EDL-5 (1984) 71.

[31] Katto H., OkuYama K., Meguro S., Nagai N. et Ikeda S., IEDM Tech. Digest (1984) 774.

[32] Toyoshima Y., Nihira H., Wada M. et KanZaKi K., IEDM Tech. Digest (1984) 786.

[33] Doyle B. S., Bourcerie M., Bergonzoni C., Bennechi R., Bravaix A., Mistry K. R. et Boudou A., IEEE Trans. Electron. Dev. 37 (1990) 1869.

[34] DOYLe B. S. et MistRY K. R., IEEE Electron. Dev. Lett. 12 (1991) 178.

[35] Weber W., WeRner C, et DORDA G., IEEE Electron. Dev. Lett. EDL-5 (1984) 518.

[36] Choi J. Y., Ko P. K. et Hu C., IEEE Electron. Dev. Lett. EDL-8 (1987) 333.

[37] Bellens R., Heremans P., Groeseneken G. et Maes H. E., IEDM Tech. Digest (1988) 212.

[38] WeBer W., IEEE Trans. Electron. Dev. 35 (1988) 1476.

[39] Bellens R., Heremans P., Groeseneken G. et Maes H. E., IEEE Electron. Dev. Lett. 9 (1988) 232.

[40] Jupina M. A. et Lenahan P. M., IEEE Trans. Nuclear Sci. 36 (1989) 1800.

[41] Jupina M. A. et Lenahan P. M., IEEE Trans. Nuclear Sci. 37 (1990) 1650.

[42] Krick J. T., Lenahan P. M. et Dunn G. J., Appl. Phys. Lett. 59 (1991) 3437.

[43] Vuillaume D., Marchetaux J. C. et Boudou A., IEEE Electron. Dev. Lett. 12 (1991) 60.

[44] Marchetaux J. C., Vullaume D. et Boudou A., IEEE Electron. Dev. Lett. 11 (1990) 406.

[45] Bourcerie M., Marchetaux J. C., Boudou A. et Vuillaume D., Proc. essderC'89, A. Henberger, H. Ryssel et P. Lange Eds. (Springer Verlag, Berlin, 1989) p. 549.

[46] Bourcerie M., Marchetaux J. C., Boudou A. et Vuillaume D., Appl. Phys. Lett. 55 (1989) 2193.

[47] Vulllaume D. et Doyle B. S., Solid State Electron. (1992) accepté.

[48] Vuillaume D., 181st Electrochem. Soc. Meeting, 2nd symposium on physics and chemistry of $\mathrm{SiO}_{2}$ and $\mathrm{Si}_{-} \mathrm{SiO}_{2}$ interface (St Louis, Missouri, 1992), accepté.

[49] Vuillaume D., Marchetaux J. C., Lippens P. E., BravaiX A. et Boudou A., IEeE Trans. Electron. Dev., soumis.

[50] Doyle B. S., Faricelli J., Mistry K. R. et Vutllaume D., IEEE Electron. Dev. Lett., soumis.

[51] Collard D. et Taniguchi T., IEEE Trans. Electron. Dev. 33 (1986) 1454.

[52] Collard D., Baccus B., Dubois E. et Morel D., « Software tools for Process, devices and circuit modelling », W. Crans Ed. (Boole Press, Dublin, 1989) p. 16.

[53] LIPPENS P., Compte-rendu de contrat BULL-ISEN-CNRS, non publié.

[54] Dubors E., Baccus B., Collard D. et Morel D., Notice d'utilisation du logiciel IMPACT 3.3 (janv. 91), non publié.

[55] Saks N. S., Heremans P. L., van den Hove L., Maes H. E., DeKeersmaecker R. F. et DeClerCK G. J., IEEE Trans. Electron. Dev. ED-33 (1986) 1529.

[56] Gaensslen F. H. et AItKen J. M., IEEE Electron. Dev. Lett. EDL-1 (1980) 231.

[57] NisSAN-COHEN Y., IEEE Electron. Dev. Lett. EDL-7 (1986) 561.

[58] TAM S., Ko P. K. et Hu C., IEEE Trans. Electron. Dev. ED-31 (1984) 1116.

[59] Heremans P., Witters J., Groeseneken G. et MaEs H. E., IEEE Trans. Electron. Dev. ED-36 (1989) 1318.

[60] Doyle B. S. et MistRy K. R., IEEE Trans. Electron. Dev., soumis.

[61] Dimaria D. J., « The Physics of $\mathrm{SiO}_{2}$ and its interface», S. T. Pantelides Ed. (Pergamon, New York, 1978) p. 160.

[62] Lannoo M., Proc. 13th Int. Conf. on Defects in Semiconductors, L. C. Kimmerling et J. M. Parsey Eds. (The Metallurgical Soc. of AIME, Warrendale, 1984) p. 221.

[63] Brugler J. S. et Jespers P. G., IEEE Trans. Electron. Dev. ED-16 (1969) 297.

[64] Heremans P., Maes H. E. et Saks N., IEEE Electron. Dev. Lett. EDL-7 (1986) 428.

[65] Ancona M. G., Saks N. S. et MCCarthy D., IEEE Trans. Electron. Dev. 35 (1988) 2221.

[66] PoOle H. H., Philos. Mag. (London) 33 (1916) 112.

[67] Frenkel J., Phys. Rev. 54 (1938) 647. 
[68] Bourgoin J. C. et Lannoo M., « Point defects in semiconductors » 2 (Springer, Berlin, 1983) p. 199.

[69] Buchwald W. R. et Johnson N. M., J. Appl. Phys. 64 (1988) 958.

[70] LANNOO M., communication privée.

[71] Bourgoin J. C. et LanNoo M., « Point defects in semiconductors » 2 (Springer, Berlin, 1983) pp. 88-121.

[72] Goguenheim D. et Lannoo M., J. Appl. Phys. 68 (1990) 1059.

[73] GRISCOM D. L., J. Non-Cryst. Solids 73 (1985) 51.

[74] Voir une revue dans : GrIscom D. L., The Physics of $\mathrm{SiO}_{2}$ and its interfaces, S. T. Pantelides Ed. (Pergamon, New York, 1978) p. 232 ;

Griscom D. L., Brown D. B. et SAKs N. S., The Physics and Chemistry of $\mathrm{SiO}_{2}$ and the $\mathrm{Si}^{-\mathrm{SiO}_{2}}$ interface, C. R. Helms et B. E. Deal Eds. (Plenum, New York, 1988) p. 287.

[75] Griscom D. L., Phys. Rev. B 20 (1979) 1823.

[76] Griscom D. L., Phys. Rev. B 22 (1980) 4192.

[77] Griscom D. L. et Friebele E. F., Phys. Rev, B 24 (1981) 4896.

[78] O'Reilly E. P. et Robertson J., Phys. Rev. B 27 (1983) 3780.

[79] RoBertson J., Philos. Mag. B 52 (1985) 371.

[80] Rong F., Poindexter E. H., Harmatz M., Buchwald W. R. et Gerardi G. J., Solid State Commun. 76 (1990) 1083.

[81] Vranch R. L., Henderson B. et Pepper M., Appl. Phys. Lett. 52 (1988) 1161.

[82] Christmann P., Bernauer M., Wetzel C., Asenov A., Meyer B. K. et Endrös A., Proc. ICDS16, Eds. G. Davies, G. G. Deheo et M. Stavola (Trans. Tech. Publications, Zürich, 1991) p. 1165 .

[83] Rong F. C., Buchwald W. R., Poindexter E. H., Warren W. L. et Keeble D. J., Solid-State Electron. 34 (1991) 835.

[84] Artken J. M. et Young D. R., IEEE Trans. Nucl. Sci. 24 (1977) 2128.

[85] Hui J., Hsu F. C. et Moll J., IEEE Electron. Dev. Lett. EDL-6 (1985) 135.

[86] Ancona M. G. et Saks N. S., Proc. 6th NASCODE Conf. (1989) p. 415.

[87] Heyns M. M., Krishna-Rao D. et DeKeersmaecker R. F., Appl. Surf. Sci. 39 (1990) 237.

[88] YEOW Y. T., LiNg C. H. et AH L. K., IEEE Electron. Dev. Lett. 12 (1991) 366.

[89] Fang P., Ko P. K. et Hu C., IEEE Electron. Dev. Lett. 12 (1991) 273.

[90] Bravaix A., Boudou A. et Vullaume D., IEEE Trans. Electron. Dev., soumis.

[91] BravaiX A., Thèse de Doctorat en micro-électronique de l'Université Paris VII (octobre 1991) non publié. 